\title{
Faranoff-Riley type I jet deceleration at density discontinuities
}

\section{Relativistic hydrodynamics with a realistic equation of state ${ }^{\star}$}

\author{
Z. Meliani ${ }^{3}$, R. Keppens ${ }^{1,3,4}$, and B. Giacomazzo ${ }^{2}$ \\ 1 FOM-Institute for Plasma Physics Rijnhuizen, Nieuwegein \\ 2 Max-Planck-Institut für Gravitationsphysik, Albert-Einstein-Institut, Golm, Germany \\ 3 Centre for Plasma Astrophysics, KU Leuven (Leuven Mathematical Modeling and Computational Science Center), Belgium \\ e-mail: [Zakaria.Meliani ; Rony.Keppens]@wis . kuleuven . be \\ 4 Astronomical Institute, Utrecht University, The Netherlands
}

Received 4 December 2007 / Accepted 14 August 2008

\begin{abstract}
Aims. We propose a model that could explain the sudden jet deceleration in active galactic nuclei, thereby invoking density discontinuities. Motivated particularly by recent indications from HYbrid MOrphology Radio Sources (HYMORS) that Fanaroff-Riley classification is induced in some cases by variations in the density of the external medium. We explore how one-sided jet deceleration and a transition to FR I type can occur in HYMORS, which start as FR II (and remain so on the other side).

Methods. We implemented the Synge-type equation of state introduced in the general polytropic case by Meliani et al. (2004, A\&A, $425,773)$ into the relativistic hydrodynamic grid-adaptive AMRVAC code. To demonstrate its accuracy, we set up various test problems in an appendix, which we compare to exact solutions that we calculate as well. We use the code to analyse the deceleration of jets in FR II/FR I radio galaxies, following them at high resolution across several hundred jet beam radii.

Results. We present results for 10 model computations that vary the inlet Lorentz factor from 10 to 20, include uniform or decreasing density profiles, and allow for cylindrical versus conical jet models. As long as the jet propagates through uniform media, we find that the density contrast sets most of the propagation characteristics, fully consistent with previous modelling efforts. When the jet runs into a denser medium, we find a clear distinction in the decelaration of high-energy jets depending on the encountered density jump. For fairly high-density contrast, the jet becomes destabilised and compressed, decelerates strongly (up to subrelativistic speeds), and can form knots. If the density contrast is too weak, the high-energy jets continue with FR II characteristics. The trend is similar for the low-energy jet models, which start as underdense jets from the outset, and decelerate by entrainment into the lower region as well. We point out differences that are found between cylindrical and conical jet models, together with dynamical details like the Richtmyer-Meshkov instabilities developing at the original contact interface.
\end{abstract}

Key words. hydrodynamics - galaxies: jets - ISM: jets and outflows - gamma rays: bursts

\section{Introduction}

Accretion discs surrounding black holes, jets found in association with compact objects, and gamma ray bursts (GRBs) all represent violent astrophysical phenomena. They are associated with relativistic flows, both with respect to the occurring velocities and to their prevailing equation of state. The jets from active galactic nuclei (AGN) and in GRBs are accelerated in a short distance to reach a high Lorentz factor: typical values are $\gamma \sim 5-30$ (Kellermann et al. 2004) for AGNs and $\gamma>100$ for GRBs (Woods \& Loeb 1995; Sari \& Piran 1995). In this acceleration phase, situated at the base of the jet, it is believed that jet energy is dominated by thermal energy and Poynting flux and that a fraction of these energies contributes to the efficient acceleration of the flow. Becaus of this, the relativistic fluid changes its state from relativistic, corresponding to an effective polytropic index $\Gamma=4 / 3$, to classical (polytropic index $\Gamma=5 / 3$ ) when the thermal energy is converted to kinetic energy. Farther along the jet paths, where the jets interact with the surrounding medium, a fraction of the directed kinetic energy is also converted into thermal energy as the shock fronts formed. According to the

\footnotetext{
* Appendices are only available in electronic form at http: //www . aanda.org
}

prevailing Lorentz factor of the jet, these shocks could be relativistic and therefore very efficient for converting its kinetic into thermal energy, or could be Newtonian and only weakly efficient in compression. To investigate these relativistic flows, a realistic equation of state must therefore be adopted to handle both classical and relativistic temperature variations in space and time. For that purpose, we implemented the Synge-type equation of state introduced for a general polytropic case as in Meliani et al. (2004) into the relativistic (magneto)hydrodynamic gridadaptive AMRVAC code (Meliani et al. 2007; Keppens et al. 2003; van der Holst \& Keppens 2007) as applied in the adiabatic case by Mignone \& McKinney (2007). To demonstrate its accuracy, we set up various stringent test problems in an appendix, which we compare to exact solutions for Riemann problems, which we calculate as well. The latter include Riemann problems at Lorentz factors of order $\gamma \approx 100$, which represent extreme values relevant for about GRB flows.

The development of relativistic numerical hydrodynamic codes can help us understand the physics of astrophysical jet propagation. In the last decade, significant progress was made in numerical special relativistic hydrodynamic (HD) and magnetohydrodynamic codes. Various authors worked on the development of conservative shock-capturing schemes which use 
based on either exact or approximate Riemann solver methods for relativistic hydrodynamics (Eulderink \& Mellema 1994; Font et al. 1994; Aloy et al. 1999; Del Zanna \& Bucciantini 2002; Mignone \& Bodo 2005) (for a review see Martí \& Müller 2003). The study of relativistic hydrodynamic fluids also benefits from spatial and temporal adaptive techniques, or adaptive mesh refinement (Duncan \& Hughes 1994; Zhang \& MacFadyen 2006; Meliani et al. 2007; Wang et al. 2007). The various numerical simulations usually involve a simplified equation of state (EOS) with a constant polytropic index. Notable exceptions with more realistic EOS treatments are found in Mignone et al. (2005); Mignone \& McKinney (2007); Perucho \& Martí (2007). We present the required extension of the AMRVAC code (Meliani et al. 2007; Keppens et al. 2003; van der Holst \& Keppens 2007) in the Appendix to this paper with the more realistic polytropic EOS introduced by Meliani et al. (2004), which is based on the Synge gas equation, the relativistically correct perfect gas law (Synge 1957; Mathews 1971). The equations we solve and the schemes used are also mentioned there.

Many previous investigations of relativistic jet propagation through the interstellar medium (ISM) in radio galaxies concentrate on uniform ISM conditions (Duncan \& Hughes 1994; Martí et al. 1997; Komissarov \& Falle 1998; Aloy et al. 1999). These investigations have contributed to our understanding of the jet deceleration, where then distinguishe the dynamics for Faranoff-Riley type I (FR I) and FR II radio galaxies, according to the power of the jet and hence the accretion rate in their galactic centre. In the FR I radio galaxies, the associated jets are relativistic on parsec scales and sub-relativistic on kiloparsec-scales, so that jet deceleration, hence energy redistributions, must happen on kiloparsec scales (Hardcastle et al. 2005). Various studies have looked into possible deceleration processes with both non-relativistic and relativistic HD codes. Hooda \& Wiita (1996, 1998) used with a classical HD code to investigate the propagation of a 3D jet through an interface between dense ISM and less dense intercluster medium. They found that, for such interface marking a density decrease, the jet does not decelerate when crossing it and that the deceleration mostly happens in the lower region (dense ISM). Moreover, the jets are not deflected at the interface ISM/ICM in their study. Norman et al. (1988) also used a classical code to study the sudden deceleration of jets, when the jet crosses a shock wave in the external medium. Other works have investigates the interaction of jets with dense clouds in both non-relativistic hydrodynamic simulations (Saxton et al. 2005) and relativistic simulations (Choi et al. 2007). Duncan et al. (1996) studied relativistic jet propagation in uniform overdense media and used an equation of state for a pair-plasma. Scheck et al. (2002) study the influence of the matter composition of a high jet energy jet on its interaction with the external medium. They investigate the two extreme cases of pure leptonic and baryonic plasmas. Perucho \& Martí (2007) examined the propagation of the low energy jet of the specific FR I radio source 3C 31 using an elaborate hydrodynamics model, with an equation of state that distinguishes the contribution of leptons and baryons to density and pressure. Most recently, Rossi et al. (2008) investigate the propagation of jets in uniform overdense media in $3 \mathrm{D}$, using a realistic synge EOS.

In this paper, we investigate a scenario of relativistic jet propagation through an ISM with a sudden jump in density. Since jets propagate over enormous distances, it is inevitable that they encounter density jumps in the external medium. As we assumed that matter in the inner part of the radio galaxy has been cleared away during the evolution of the radio galaxy, we typically followed jet dynamics through a lighter medium at first, which then suddenly changes far away to a denser medium. In a sequence of model runs, we varied the jet and medium properties to cover both high and low jet beam kinetic luminosities, straight as well as conical jets (varying the jet opening angle), and allowed for either uniform or decreasing density profiles. To put our work into perspective and to appreciate its relation to previous works better, we compiled in Table 1 the most relevant parameters and simulation specifics for selected relativistic hydro models.

The most novel assumption is the transition from low to high density across a contact interface (across which the pressure is necessarily constant), which is different from the work done by Hooda \& Wiita (1998) where they used a classical code with a density decrease across the contact, and also different from the study by Loken et al. (1993), where they used a classical code to look into jet propagation through a pressure wall. We explored the influence of a sudden density jump in ISM on jet propagation, stability, and formation of the bow shock. We aim to better understand the jet efficiency in transporting energy and mass from the central AGN regions to the denser ISM at large distances, where the jet cocoon is formed. From Table 1 it is clear that our models extend the previous works most notably by typically covering much larger distances than previously studied (as we need to obtain representative endstate morphologies in both lower and upper media), and are invariably in the high Lorentz factor regime, combined with high resolution through the jet beam. The high-energy jets we consider start out as denser than their immediate surroundings (a likely property deduced from all common jet launch scenarios, but previously ignored by all jet simulations to date). Moreover, the density contrasts we investigate between jet and outer medium are much more reasonable than the 5 orders of magnitude density differences previously used by Scheck et al. (2002). In what follows, we first motivate our model assumptions and the simulation setup in Sect. 2. We discuss our main findings in Sect. 3.

\section{Relativistic jet propagation and deceleration}

The radio-loud Fanaroff-Riley galaxies (Fanaroff \& Riley 1974) have extensively been studied in the last decade, because their jets show intriguing behaviour. In fact, radio loud galaxies are grouped into two main classes according to their radio map morphology. A typical FR I is brighter near the centre and fades out towards the edge, whereas FR II are brightest at the edges and fainter toward the centre. Fanaroff \& Riley (1974) discovered that the break in FR I/FR II occurs around the radio luminosity of $P_{178 \mathrm{MHz}} \sim 10^{25} \mathrm{~W} \mathrm{~Hz}^{-1} \mathrm{sr}^{-1}$, with almost all sources below the break value being of type FR I. In contrast to FR II galaxies where the jet remains relativistic and narrow on all scales, the jet in FR I (Giovannini et al. 2005) is relativistic on the parsec scale (Bridle 1992) and in many cases subrelativistic and diffuse on kparsec-scales (Giovannini et al. 2001). The FR I jet shows side-to-side asymmetries that decrease with distance from the central engine, caused by the decrease in Doppler beaming in an intrinsically symmetrical, decelerating jet (Laing et al. 1999). However, in some FR I the structure of the jet in the kiloparsec scale appears more complicated, with an inner spine that remains relativistic and an outer shell that decelerates and becomes subrelativistic (Canvin et al. 2005).

It is commonly accepted that the morphological differences between FR I/FR II arise because of differences in the physical conditions of jet interaction with its environment. However, it is still under debate whether the observed deceleration in FR I jets is related to the mechanism of jet launching and thus to the properties of the central engine in AGN (Ghisellini \& Celotti 2001; 
Table 1. The most relevant characteristics and parameters for various selected relativistic hydrodynamic simulations in the literature. We mention the type of the simulation 2D or 3D and use of AMR, jet beam kinetic luminosity $L_{\mathrm{b}}$ (when available), $\gamma_{\mathrm{b}}$ Lorentz factor of the beam, $M_{\mathrm{b}}$ relativistic Mach number, $\eta$ density ratio ("d" means density variation), $\theta$ open angle of the jet, $R_{\mathrm{b}} / r_{\text {cell }}$ grid cells through jet beam, type of EOS ("Lep" means Leptonic, "Bar" Baryonic), endtime of the simulation in units of light crossing time of the jet beam radius.

\begin{tabular}{|c|c|c|c|c|c|c|c|c|c|}
\hline Paper $^{a}$ & case & $\overline{L_{\mathrm{b}}}$ & $\gamma_{\mathrm{b}}$ & $\overline{M_{\mathrm{b}}}$ & $\overline{\rho_{\text {medium }} / \rho_{\text {jet }}}$ & $\overline{\theta_{\mathrm{b}}}$ & $\bar{R} R_{\mathrm{b}} / r_{\text {cell }}$ & $\overline{\mathrm{EOS}}$ & Size in $R_{\mathrm{b}}$ (and time) \\
\hline DH94/R99 & $\mathrm{A}(2 \mathrm{D}, \mathrm{AMR})$ & - & 1.048 & 6 & 10.0 & 0 & 24 & $5 / 3$ & $10 \times 41.67(-)$ \\
\hline DH94/R99 & $\mathrm{B}(2 \mathrm{D}, \mathrm{AMR})$ & - & 5.0 & 8 & 10.0 & 0 & 24 & $5 / 3$ & $16.67 \times 41.67(-)$ \\
\hline DH94/R99 & $\mathrm{C}(2 \mathrm{D}, \mathrm{AMR})$ & - & 10.0 & 8 & 10.0 & 0 & 24 & $5 / 3$ & $16.67 \times 41.67(-)$ \\
\hline DH94/R99 & $\mathrm{D}(2 \mathrm{D}, \mathrm{AMR})$ & - & 10.0 & 15 & 10.0 & 0 & 24 & $4 / 3$ & $16.67 \times 41.67(-)$ \\
\hline M 97 & A1 (2D) & - & 7.1 & 9.97 & $10^{2}$ & 0 & 20 & $4 / 3$ & $10.5 \times 50(50)$ \\
\hline M 97 & A2 (2D) & - & 22.37 & 31.86 & $10^{2}$ & 0 & 20 & $4 / 3$ & $10.5 \times 50(50)$ \\
\hline M 97 & a1 (2D) & - & 7.1 & 9.97 & 1.0 & 0 & 20 & $4 / 3$ & $10.5 \times 25(25)$ \\
\hline M 97 & a2 (2D) & - & 7.1 & 9.97 & 10.0 & 0 & 20 & $4 / 3$ & $10.5 \times 25(25)$ \\
\hline M 97 & B1 (2D) & - & 7.1 & 41.95 & $10^{2}$ & 0 & 20 & $4 / 3$ & $10.5 \times 50(50)$ \\
\hline M 97 & B2 (2D) & - & 22.37 & 132.32 & $10^{2}$ & 0 & 20 & $4 / 3$ & $10.5 \times 50(50)$ \\
\hline M 97 & b1 (2D) & - & 2.29 & 13.61 & 10.0 & 0 & 20 & $4 / 3$ & $10.5 \times 25(25)$ \\
\hline M 97 & b2 (2D) & - & 7.1 & 41.95 & 10.0 & 0 & 20 & $4 / 3$ & $10.5 \times 25(25)$ \\
\hline M 97 & C1 (2D) & - & 2.29 & 13.61 & $10^{2}$ & 0 & 20 & $5 / 3$ & $10.5 \times 50(50)$ \\
\hline M 97 & $\mathrm{C} 2(2 \mathrm{D})$ & - & 7.1 & 41.95 & $10^{2}$ & 0 & 20 & $5 / 3$ & $10.5 \times 50(50)$ \\
\hline M 97 & C3 (2D) & - & 22.37 & 132.32 & $10^{2}$ & 0 & 20 & $5 / 3$ & $10.5 \times 50(50)$ \\
\hline M 97 & c1 (2D) & - & 2.29 & 13.61 & 10.0 & 0 & 20 & $5 / 3$ & $10.5 \times 25(25)$ \\
\hline M 97 & c2 (2D) & - & 7.1 & 41.95 & 10.0 & 0 & 20 & $5 / 3$ & $10.5 \times 25(25)$ \\
\hline H98 & $\mathrm{B}(2 \mathrm{D}, \mathrm{AMR})$ & - & 5.52 & 8.87 & 10.0 & 0 & 24 & $5 / 3$ & $16.67 \times 41.67(140.0)$ \\
\hline H98 & $\mathrm{C}(2 \mathrm{D}, \mathrm{AMR})$ & - & 14.35 & 11.52 & 10.0 & 0 & 24 & $5 / 3$ & $16.67 \times 41.67(21.43)$ \\
\hline H98 & $\mathrm{D}(2 \mathrm{D}, \mathrm{AMR})$ & - & 10.0 & 15.0 & 10.0 & 0 & 24 & $4 / 3$ & $16.67 \times 41.67(35.273)$ \\
\hline H98 & $\mathrm{E}(2 \mathrm{D}, \mathrm{AMR})$ & - & 2.55 & 8.53 & 10.0 & 0 & 24 & $5 / 3$ & $16.67 \times 41.67(140.0)$ \\
\hline S02 & $\mathrm{A}(2 \mathrm{D})$ & $10^{46}$ & 6.62 & 9.24 & $10^{5}$ & 0 & 6 & Lep & $200 \times 500(5950)$ \\
\hline S02 & $\mathrm{B}(2 \mathrm{D})$ & $10^{46}$ & 6.62 & 14.33 & $10^{3}$ & 0 & 6 & Lep & $200 \times 500(5400)$ \\
\hline S02 & $C(2 D)$ & $10^{46}$ & 7.95 & 130.14 & $10^{3}$ & 0 & 6 & Bar & $200 \times 500(5300)$ \\
\hline PM07 & $\mathrm{A}(2 \mathrm{D})$ & $10^{44}$ & 2.0 & 4.75 & $10^{5}$ “d" & 0 & 16 & Lep/Bar & $200 \times 450(37231)$ \\
\hline R08 & $\mathrm{A}(3 \mathrm{D})$ & - & 10.0 & 28.3 & $10^{2}$ & 0 & 20 & Synge & $50 \times 150 \times 50(240)$ \\
\hline R08 & $\mathrm{B}(3 \mathrm{D})$ & - & 10.0 & 28.3 & $10^{4}$ & 0 & 20 & Synge & $60 \times 75 \times 60(760)$ \\
\hline R08 & $\mathrm{C}(3 \mathrm{D})$ & - & 10.0 & 28.3 & $10^{4}$ & 0 & 12 & Synge & $50 \times 75 \times 50(760)$ \\
\hline R08 & $\mathrm{D}(3 \mathrm{D})$ & - & 10.0 & 300 & $10^{4}$ & 0 & 20 & Synge & $50 \times 150 \times 50(760)$ \\
\hline R08 & $E(3 D)$ & - & 10.0 & 300 & $10^{2}$ & 0 & 12 & Synge & $24 \times 200 \times 24(150)$ \\
\hline MKG & $\mathrm{A}(2 \mathrm{D}, \mathrm{AMR})$ & $10^{46}$ & 20.0 & 1200 & $0.1496-4.687$ & 0 & 64 & Synge & $10 \times 400$ \\
\hline $\mathrm{MKG}$ & $\mathrm{B}(2 \mathrm{D}, \mathrm{AMR})$ & $10^{46}$ & 20.0 & 1200 & $0.1496-671.22$ & 0 & 128 & Synge & $10 \times 400(900)$ \\
\hline $\mathrm{MKG}$ & $\mathrm{C}(2 \mathrm{D}, \mathrm{AMR})$ & $10^{43}$ & 10.0 & 39 & 36.52-203.66 “d” & 0 & 76 & Synge & $10 \times 400(820)$ \\
\hline MKG & $\mathrm{D}(2 \mathrm{D}, \mathrm{AMR})$ & $10^{43}$ & 10.0 & 39 & $36.52-148.15$ "d" & 1 & 76 & Synge & $10 \times 400(820)$ \\
\hline MKG & $\mathrm{E}(2 \mathrm{D}, \mathrm{AMR})$ & $10^{43}$ & 20.0 & 35 & 146-847.46 “d” & 0 & 76 & Synge & $40 \times 400(820)$ \\
\hline MKG & $\mathrm{F}(2 \mathrm{D}, \mathrm{AMR})$ & $10^{43}$ & 20.0 & 35 & 146-645.16 "d" & 1 & 76 & Synge & $40 \times 400(820)$ \\
\hline MKG & $\mathrm{G}(2 \mathrm{D}, \mathrm{AMR})$ & $10^{46}$ & 10.0 & 1300 & $0.033-0.495$ "d" & 0 & 144 & Synge & $40 \times 400(380)$ \\
\hline MKG & $\mathrm{H}(2 \mathrm{D}, \mathrm{AMR})$ & $10^{46}$ & 10.0 & 1300 & $0.033-30.6748$ “d” & 1 & 76 & Synge & $40 \times 400(800)$ \\
\hline MKG & $\mathrm{I}(2 \mathrm{D}, \mathrm{AMR})$ & $10^{46}$ & 10.0 & 1300 & $0.033-0.306748$ "d" & 1 & 76 & Synge & $40 \times 400(480)$ \\
\hline MKG & $\mathrm{J}(2 \mathrm{D}, \mathrm{AMR})$ & $10^{46}$ & 20.0 & 1200 & $0.1496-12.95$ “d” & 1 & 76 & Synge & $40 \times 400(480)$ \\
\hline
\end{tabular}

${ }^{a}$ DH94 (Duncan \& Hughes 1994), M 97 (Martí et al. 1997), H98 (Hardee et al. 1998), R99 (Rosen et al. 1999) S02 (Scheck et al. 2002$)$, PM07 (Perucho \& Martí 2007), R08 (Rossi et al. 2008). Our simulations are indicated by MKG.

Kaiser \& Best 2007), or rather related to the properties of the external medium: the host galaxy (Zirbel 1997) and the circumgalactic gas (De Young 1993; Kaiser \& Alexander 1997). Some authors Wold et al. (2007) claim that the FR I/FR II dichotomy is caused by the combination of a central engine factor, with a contribution of the external medium. Observational evidence that the external medium influence comes from the existence of a group of peculiar "HYbrid MOrphology Radio Source" (HYMORS), pointed out by Gopal-Krishna \& Wiita $(2000,2002)$. These radio sources appear to have an FR II type on one side and an FR I type diffuse radio lobe on the other side of the active nucleus. This supports the idea that the different Fanaroff-Riley morphologies are attributed in some cases to the properties of the ambient media, since for HYMORS, similar jets (power, composition, Lorentz factor) likely emerge from the central engine on each side. Heywood et al. (2007) have analysed radio images for a set of quasars 7C (radiosource galaxies) and find that some FR I seem to have a radio luminosity exceeding the original FR I/FR II dividing line. They also confirmed the existence of HYMORS and suggested that these sources have high-power jets (typical for FR II) and they yield FR I or FR II morphologies according to their external medium. In a rarefied medium, the jet is "laminar" and remains collimated all the way to the intergalactic medium (IGM) where the hotspot forms (the impact site of the jet in the IGM). This gives an FR II jet morphology with the typical "lobe". In dense or clumpy medium, the jet interacts strongly with its external medium and dissipates its energy more gradually as it propagates in this dense medium. This gives the FR I jet morphology with the typical "plume" on large spatial scales.

We point out several concrete examples next. The bright onesided (FR I-like), diffuse jet in the radio galaxy 3C 321 (which is an FR II radio source) is an excellent example of a HYMORS. Evans et al. (2008) argue that the one-sided diffuse jet is the 
result of an interaction of the jet with the companion galaxy. That both sides of the jets are relativistic and stable on the parsec scale in these objects, and the difference appears on the large scale means that the variation in the external medium must occur at some distance from the central engine. This is what we assume in our models. The radio galaxy Cen A shows also a difference in the radio morphology between the two sides of the jet as it propagates on kiloparsec scales, with edge-brightened lobes (FR I-like) on one side, and on the other side a central (fine structured) lobe (FR II-like) (Kraft et al. 2003). Yet another example is the powerful radio source Hercules A (3C 348) that has a jet kinetic luminosity $10^{46} \mathrm{ergs} / \mathrm{s}$ (McNamara et al. 2005) and exhibits a mixed FR I/FR II morphology (Sadun \& Morrison 2002). These observations confirm the idea that, in Cen A and Hercules A (3C 348), the external medium plays a key role in the appearance of the jet and its dynamics. There is also evidence of disruptions or variations in radio morphologies induced by inhomogeneous medium; for example, the evident hierarchical structure of M 87 in its radio image (Owen et al. 2000), with the possibility of the existence of two haloes: an inner halo that could be with more porous structure, while the jet interacts mainly with the outer halo (Owen et al. 2000). In conclusion, some FR I jets propagate through clumpy (Croft et al. 2006) or dense media, evidently encountering sudden density changes. This is known to give rise to a strong interaction, and the jet loses its energy by entrainment and diffusion (De Young 1993, 1996; Rosen \& Hardee 2000; Tavecchio et al. 2006) and also forms knots along the jet (Owen et al. 1989). We now explain our new, more elaborate model, with which we aim to explain observations of jets in radio galaxies that are relativistic on a small scale and decelerate to sub-relativistic velocities approaching the large scale.

\subsection{Model}

To make the problem more tractable, we assume axisymmetry and neglect the influence of the magnetic field in the dynamics. In our scenario, we assume the existence of a density jump in the host galaxy or in circumgalactic gas, which could be responsible for the jet deceleration and knot formation in the asymptotic region. Since the jets travel enormous distances, it is inevitable that they encounter various sudden transitions of interstellar medium properties. These could be traveling shock fronts, more gradually varying background variations as one traverses regions of differing gravitational potential, or contact discontinuities indicative of boundaries between varying regions of influence. We concentrate on the latter, representing density (and entropy) changes across which total force balance holds (uniform pressure), as these are invariably found in any kind of hydrodynamic interaction involving winds, outflows, etc.

We model jet propagation through two distinct media. In the first part, a low and uniform density is assumed, such that the jet-external medium interaction is weak in this region. We will typically consider jets that are denser than the lower surrounding medium (but also include models where the jet is already underdense in this region). If the external medium in the inner region is denser than the jet, one expects strong disruption of the jet and hence diffuse and destabilised jets in this inner (parsec scale) region where the jet would decelerate and dissipate its energy. This contrasts with observations of narrow and relativistic jets in the inner region of FR I galaxies and in the HYMORS, where the energy deposited on the large scale is comparable to the energy of the central engine (Rawlings \& Saunders 1991). In this work, we are particularly interested in radio loud FR I with a powerful jet at high Lorentz factor on the lower scale. This is the case in the HYMORS, where the jets have the same properties on small scale than the high energy FR II jets. The differences appear only on one side of the jet on the larger scale, where the jet morphology changes to show a structure characteristic of an FR I. Therefore, we assume that, further downstream, the jet encounters a high-density medium, such that the jet undergoes a strong deceleration and a strong compression. This latter part is typically the only part simulated in previous numerical jet studies, where a prescribed jet configuration penetrates a usually uniform, high-density, external medium. Moreover, we study the effect of the initial opening angle of the jet in its interaction with (layered or stratified) external medium.

For the density jump in the case of 3 C 321 , we can think of the lower region as the rarefied medium in the inter-cluster medium, while the upper region represents the denser medium of the companion galaxy. In other HYMORS, the density jump is then thought to occur on one side of the AGN where there are denser molecular clouds in the inter-cluster environment or interstellar medium. One of the main points we hereby address for the first time is the change in jet head properties during the propagation phase in the inner region, and how this in turn affects the jet stability in the upper, denser medium. Indeed, the jet interacts with the inner medium mainly through the bow shock, whereas the jet beam is only weakly disturbed laterally. The shocked swept-up matter during this phase and the shocked part of the beam, both constitute a structured bow shock ahead of the beam, and both shocked regions form a new hot layer with lower Lorentz factor, characterised by a lower Mach number. The interaction of this preformed, structured jet head with denser external medium in the outer region should produce a strong cocoon and backflow. This will also disturb the non-shocked jet beam as it penetrates the denser medium. This will increase the entrainment of ambient material through velocity shear instabilities and decelerate the jet (De Young 1993; Perucho \& Martí 2007).

\subsection{Initial conditions}

Deducing the precise internal properties for an FR I jet and its environment from observations is a difficult task, and density contrasts in particular have been obtained partly on the basis of numerical studies. However, from generic properties of jet propagation in various FR I galaxies, one can estimate the kinetic luminosity and the jet beam Lorentz factor. Then, the choice of jet and environment parameters is determined base on input from the kinetic luminosity of the jet and the estimated jet propagation speed in the two media. The kinetic luminosity of a typical powerful jet is $L_{\text {jet,Kin }} \sim 10^{46} \mathrm{ergs} / \mathrm{s}$ (Rawlings \& Saunders 1991; Daly 1995; Carilli \& Barthel 1996; Wan et al. 2000; Drake 2003; Tavecchio et al. 2004; Kino \& Takahara 2004), and we used this observationally supported value for the simulations indicated as cases A, B, G, H, I, J (see Table 1). We also investigated the propagation of low-energy jets and compared them with these more powerful jets. For more radio-quiet galaxies, a lower energy jet with $L_{\text {jet,Kin }} \sim 10^{43} \mathrm{ergs} / \mathrm{s}$ (Allen et al. 2006) is deduced, and this value is used for the simulations C, D, E, F. The integrated energy flux over the beam cross section is computed from (e.g. Bicknell \& Begelman 1996; Martí et al. 1997; Rosen et al. 1999; Scheck et al. 2002)

$L_{\mathrm{jet}, \mathrm{Kin}}=\left(\gamma_{\mathrm{b}} h_{\mathrm{b}}-1\right) \rho_{\mathrm{b}} \gamma_{\mathrm{b}} \pi R_{\mathrm{b}}^{2} v_{\mathrm{b}}$

where the subscript " $\mathrm{b}$ " indicates the jet beam, $\rho_{\mathrm{b}}$ its density, $\gamma_{\mathrm{b}}$ the Lorentz factor, $v_{\mathrm{b}}$ the speed, $\rho_{\mathrm{b}} h_{\mathrm{b}}=\rho_{\mathrm{b}}+\frac{\Gamma}{\Gamma-1} p_{\mathrm{b}}$ the enthalpy, and $R_{\mathrm{b}}$ the jet radius. For the last, when we assumed a jet with opening angle of $\theta_{\mathrm{b}}=3^{\circ}$ at $1 \mathrm{pc}$, the observed 
value in Centaurus A (Horiuchi et al. 2006), we got a jet radius $R_{\mathrm{b}} \sim 0.05$ pc. We fixed the radius $R_{\mathrm{b}}=0.05 \mathrm{pc}$ on our jet input boundary, which in turn is assumed to be located at a distance from the source of $0.5 \mathrm{pc}$.

Furthermore, an estimated propagation speed of the head of the jet can be obtained from using the expression for pressurematched jet propagation in 1D (Martí et al. 1997; Rosen et al. 1999). For a cold external medium, this yields

$v_{\text {jet }}^{1 \mathrm{D}}=\frac{\sqrt{\eta_{\mathrm{R}}}}{\sqrt{\eta_{\mathrm{R}}}+1} v_{\mathrm{b}}$

where $\eta_{\mathrm{R}}=\gamma_{\mathrm{b}}^{2} \frac{\rho_{\mathrm{b}} h_{\mathrm{b}}}{\rho_{\mathrm{m}} h_{\mathrm{m}}}$ is the ratio between the inertia in the jet and in the external medium. In the relativistic case, the inertia of the flow increases as $\gamma^{2}$ with the speed (which makes relativistic jets stabler than jets in young stellar objects). Our $t=0$ conditions then use the number density of the cold external medium in the pc-scale region set to $n_{\text {Low }}=1 \mathrm{~cm}^{-3}$ as a scaling value, together with $R_{\mathrm{b}}$ as a unit of length, in combination with $c=1$. Then, the pressure in this cold external medium is set to $p=10^{-3} m_{p} n_{\text {Low }} c^{2}$, and this value for the pressure is in fact taken to be equal in the jet, and also in the outer region that is in static equilibrium with the inner region. We take the beam Lorentz factor fixed at $\gamma_{\mathrm{b}}=20$ for the simulations A, B, E, F, J and at $\gamma_{\mathrm{b}}=10$ for the simulations C, D, G, H, I. These assumed high inlet Lorentz factors are at the observed values appropriate for FR II and BL Lac objects on pc-scale (Kellermann et al. 2004; Cohen et al. 2007). This is consistent with the hypothesis that BL Lac are FR I radio galaxies observed with a small angle to the line of sight (Urry \& Padovani 1995).

The density in the jet and, in principle, also the pressure, can be deduced using Eqs. (1) and (2), by imposing the jet head propagation Lorentz factor $\gamma_{\text {head,Low }}$ in the low-density medium (in practice, this is limited by the condition to have a positive jet pressure and density). If we choose a value $\gamma_{\text {head,Low }}=5$ $\left(v_{\text {head,Low }}=0.979796\right)$, we find a ratio between the jet beam inertia and the lower medium inertia from Eq. (2). This is in the range $\eta_{\mathrm{R}, \text { Low }}=[2672.3,3000]$ for our models with high energy $(\mathrm{A}, \mathrm{B}, \mathrm{G}, \mathrm{H}, \mathrm{I}, \mathrm{J})$, while we have $\eta_{\mathrm{R}, \text { Low }}=[1.8,2.7]$ for our models with lower energy $(\mathrm{C}, \mathrm{D}, \mathrm{E}, \mathrm{F})$. We anticipate from these inertia contrasts that the high-energy jet in the lower region is fairly stable and will conserve its narrow structure, while the lower energy jets will already be disturbed by the external medium in the lower part. When we use Eq. (1) to obtain our computational value for the density ratio between the jet beam and the external medium on the lower scale, we find $n_{\mathrm{b}} / n_{\text {Low }}=6.681$ for the simulations $\mathrm{A}, \mathrm{B}, \mathrm{J} ; n_{\mathrm{b}} / n_{\text {Low }}=29.99$ for the simulations $\mathrm{G}, \mathrm{H}, \mathrm{I} ; n_{\mathrm{b}} / n_{\text {Low }}=2.738 \times 10^{-2}$ for the simulations C, D; and finally $n_{\mathrm{b}} / n_{\text {Low }}=6.82 \times 10^{-3}$ for the simulations E, F.

For the density of the external medium in the upper, downstream region, we can argue similarly by setting a head propagation Lorentz factor on the kpc-scale. In this paper, we will investigate many cases for the upper medium conditions. In the two first cases (A, B), we choose a uniform density medium. In all other models, beyond the jump in the density at the interface between the lower and higher scale regions, the density decreases with distance from the source with a simple power law $Z_{\text {jump }} / \sqrt{R^{2}+Z^{2}}$ (Kaiser \& Alexander 1997). Furthermore, in model A, we assume that the jet undergoes weak deceleration in this upper region, where the Lorentz factor of the jet head drops to $\gamma_{\text {head,Up }}=1.5$ (mildly relativistic). Then, the ratio between the jet beam inertia and upper medium inertia is $\eta_{\mathrm{R}, \mathrm{Up}}=8.65$, which increases the influence of the external medium on the jet.
Again, similar reasoning makes it plausible to use a density ratio $n_{\mathrm{Up}} / n_{\text {jet }} \sim 4.687$ for the upper medium then. In model $\mathrm{B}$, we consider a very dense upper medium, expected to induce a strong deceleration of the jet to $\gamma_{\text {head,Up }}=1.02\left(v_{\text {head,Up }}=0.197\right)$, which corresponds to a sub-relativistic jet. The inertia ratio between the jet beam and the upper medium is in this case $\eta_{\mathrm{R}, \mathrm{Up}} \sim 0.0604$, and this very low value will lead to increasing the jet-external medium interaction and the growth of body and surface mode instabilities in the jet. We find that to end up with this low Lorentz factor in the upper high-density medium, we need to assume a fairly extreme, high value of density $n_{\mathrm{Up}} / n_{\text {jet }} \sim 671.22$. This is admittedly very high; however, many numerical simulations show that to decelerate a relativistic jet to sub-relativistic speeds, we need overdense external medium where $n_{\mathrm{Up}} / n_{\mathrm{b}}>100$ and even higher (Krause 2005). This is also seen by the exploited valued for the density contrast in most numerical simulations to date, as collected in Table 1. Furthermore, from Eq. (1) one can argue that for fixed luminosity, the jet density decreases with jet radius as $\left(0.05 \mathrm{pc} / R_{\mathrm{b}}\right)^{2}$. This suggests that, for a bigger opening angle of the jet (hence bigger jet radius), the jet density and thus also the upper external medium density will be lower, while the behaviour of the jet in this region should be the same. For all other models, the density ratio at the interface reaches the values indicated in Table 1, and then decreases outwards. To also model the effect of conical versus cylindrical jet propagation, we assume a $1^{\circ}$ opening angle at the inlet for models D, F, H, I, and $\mathrm{J}$. This opening angle is taken to be smaller than the open angle of the jet at the boundary $3^{\circ}$, since the jet is supposed to collimate slowly during its propagation.

\subsection{Employed resolution}

In all our simulations of jet propagation, we set the lower boundary at $Z_{\text {in }}=0.5\left(\frac{R_{\mathrm{b}}}{0.05 \mathrm{pc}}\right) \mathrm{pc}$ and initialise the jet material within the domain for radii $R<R_{\mathrm{b}}$ and extended to $Z=1.0\left(\frac{R_{\mathrm{b}}}{0.05 \mathrm{pc}}\right) \mathrm{pc}$. The boundary imposes a stationary mass flux at the lower boundary for radii $R<R_{\mathrm{b}}$. The lower boundary for $R>R_{\mathrm{b}}$ is open. The top boundary is set at $Z_{\text {ext }}$ and the jump in density is set at a distance $Z_{\text {jump }}=Z_{\text {ext }} / 2$, midway in our (very large) computational domain. Note again that, as is evident from Table 1 , we here cover jet propagation over distances that go as far as 400 jet beam radii and that have enough resolution to analyse with high accuracy the interaction of the jet with the external medium, and this is only feasible thanks to our AMR capabilities.

Simulation A was done on a domain with size $[R, Z] \in$ $[0,10] \times[10,400]$ (in units of $R_{\mathrm{b}}$ ), with a resolution on the base level of $40 \times 1200$. Our grid-adaptive runs allow for 5 levels, achieving an effective resolution of $640 \times 19200$. The second simulation is done on a domain with size $[R, Z] \in[0,40] \times$ $[10,400]$ and base level resolution of $160 \times 1200$; however, we allowed for 6 levels achieving an effective resolution of $57 \times$ 38400 . This anticipates that, since the ratio between the jet beam density and upper medium density is very high in the second case, the shock in this model will be very strongs, and is likely to produce a turbulent cocoon and jet in this region. All other cases are done on a domain with size $[R, Z] \in[0,40] \times[10,400]$ and with an effective resolution $3072 \times 4800$ (4 levels). All the simulations exploit the hybrid version of HLLC, as explained in the Appendix.

\section{Discussion of results}

From a basic point of view, the jet-external medium interaction is structured along the jet propagation axis in a similar way to 

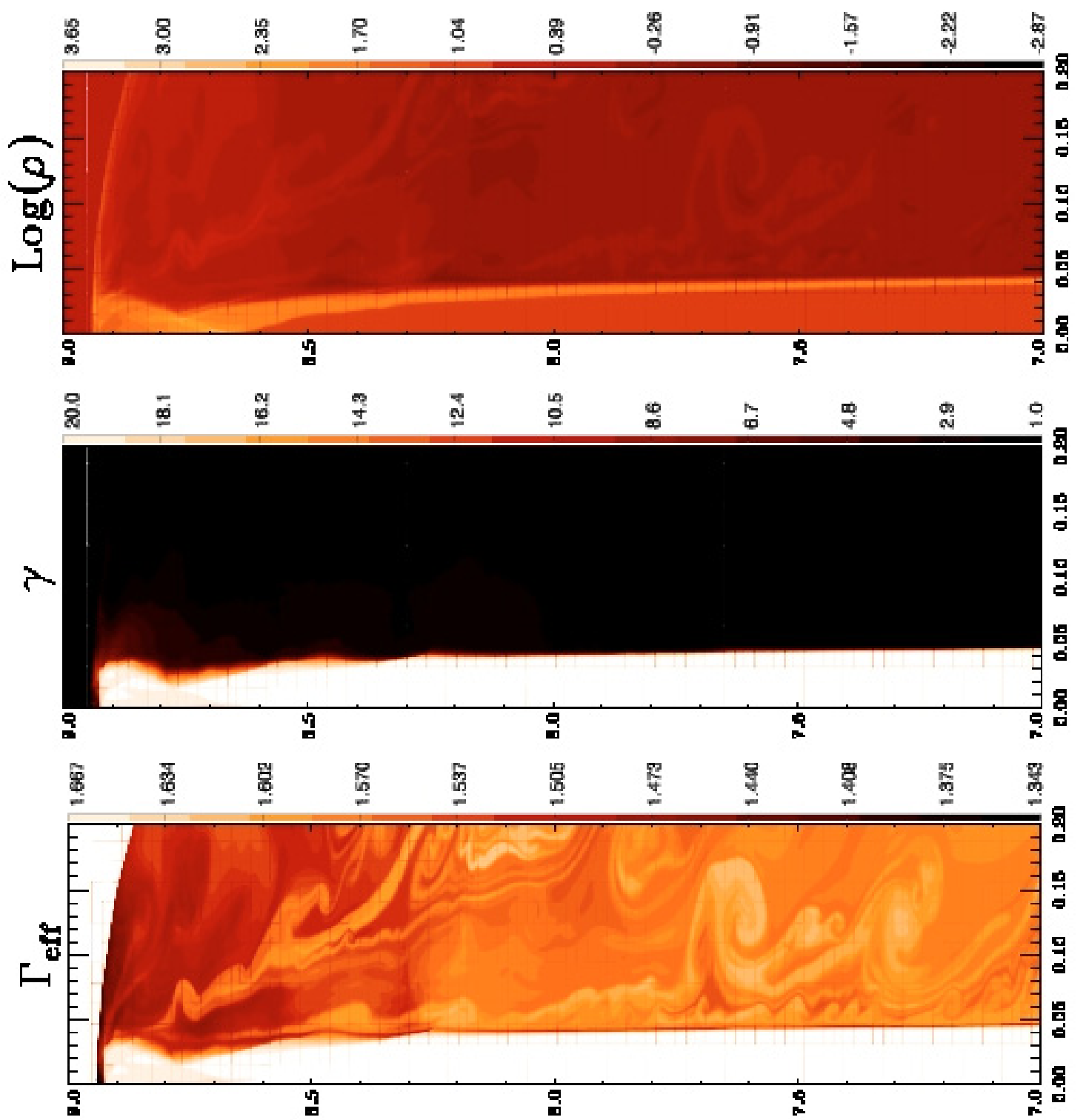

Fig. 1. A zoom on the jet head at $t=160$ light crossing times of the jet radius $R_{\mathrm{b}}$, for cases $\mathrm{A}, \mathrm{B}$, and $\mathrm{J}$, just prior to penetrating the denser upper medium. Shown are: (top) the logarithm of density, (middle) the Lorentz factor, (bottom) the effective polytropic index. In this and subsequent figures, distances indicated on the axes are shown in parsec.

the $1 \mathrm{D}$ tests described in our appendix. At the head of the jet, there are the forward shock, the contact discontinuity (called the work surface), and the reverse shock (called the Mach disc). The forward shock compresses and heats the external medium, which spreads laterally, leading to the formation of a bow shock. The reverse shock decelerates the beam matter by converting its kinetic energy into thermal energy. However, the shapes of the Mach disc and forward shock in a true 2D jet are oblique, and other $2 \mathrm{D}$ effects appear, which we describe in the following.

\subsection{Propagation through the uniform lower region}

\subsubsection{Models A, B, and J}

In models $\mathrm{A}, \mathrm{B}$, and $\mathrm{J}$, the properties of the jet and of the lower external medium are the same, and the following discussion is applicable to these 3 cases. Prior to penetrating the dense upper region the jet density, is seen in a close-up view in Fig. 1. This figure is at time $t=160$ (in units of light crossing time for the jet beam radius). In the inner, low-density medium, the forward shock is relativistic. The jet also interacts laterally with the external medium, because there is a boundary shear layer. Due to the favourable density contrast, this layer does not disturb the jet propagation in this lower region. This thin shear layer is created as the Mach disc compresses the external shell of the jet. Only near the jet head, in between working surface and reverse shock (Mach disc), and a bit beyond the reverse shock location, is the shear layer Kelvin-Helmholtz unstable (see our zoomed-in Fig. 1). We hardly find any backflow from the work surface in this lower region.

Focusing on the axial structure of the jet head, Fig. 2 compares the obtained on-axis structure with a first equivalent 1D Riemann problem. The sound speed in the shocked external medium (between forward shock and contact or work surface) increases to reach the maximum value allowed by the equation of state $c_{\mathrm{S}} \sim 0.636$; hence, the effective polytropic index in this flow drops to $\Gamma_{\text {eff }} \sim 1.34$ (a near ultra-relativistic state). Then the 

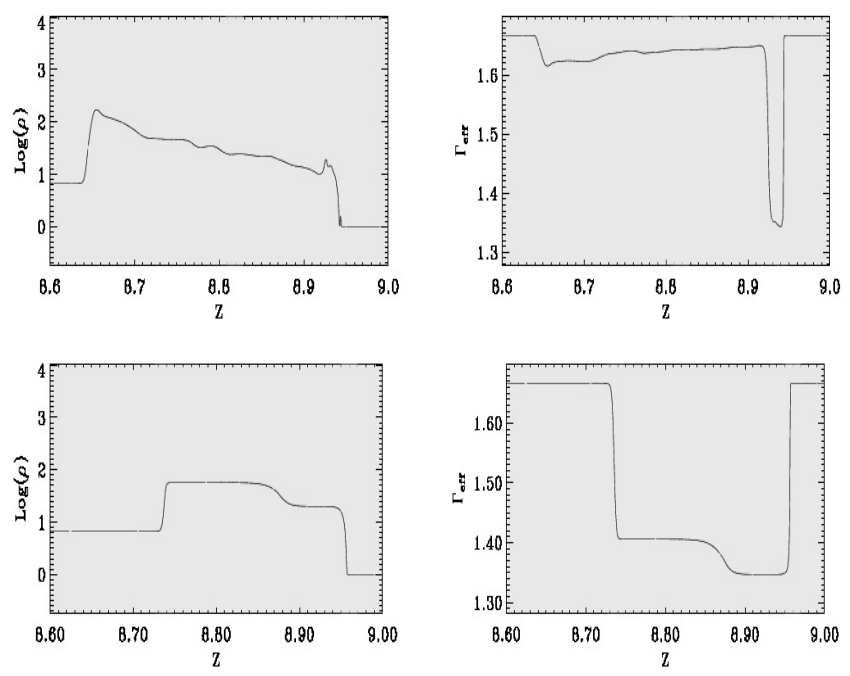

Fig. 2. Top: a cut along the $Z$ axis through the jet head at $t=160$, prior to penetrating the denser medium (cases A, B, and J). Shown is: (left) the logarithm of density, (right) the effective polytropic index. Bottom: a $1 \mathrm{D}$ equivalent relativistic shock tube problem.

compression is very high, so the distance between the contact discontinuity and forward shock remains small: we obtain a spacing of about $\delta X=0.044 \mathrm{pc}$ at $t=160$. This is even three times smaller than what was obtained in an equivalent $1 \mathrm{D}$ shock problem, as seen in Fig. 2. The difference is induced by the lateral spreading of the shocked shell of swept-up matter, which acts to decrease the amount of accreted mass. Indeed, shocked material in front of the beam will start to spread laterally, due to the strong (radial) gradient in the pressure and in the inertia between the shocked material and the cold, static external medium. This initial spreading can even be quantified from a second 1D Riemann problem in the direction across the beam (which is not shown here), and this confirms the 2D result that shows an initial supersonic lateral speed $v_{\text {lateral }} \sim 0.7973$ for the shocked material. This outward spreading leads to the formation of a bow shock, whose lateral spreading eventually (a 2D effect) decelerates to $v_{\text {lateral }} \sim 0.36$. With this fast lateral spreading of the shocked material, no complex cocoon forms in this region of low density for these three cases A, B, and J. The second equivalent Riemann problem in the radial direction also shows how a rarefaction wave propagates laterally inward into the swept-up matter by the jet. This rarefaction wave moves slowly towards the axis at approximate speed $v_{\mathrm{r}}=0.058$, and matter accumulates mainly near the jet axis. This produces relatively more deceleration of the jet near the axis, hence to a radially structured jet head.

Returning to the axial structure as shown in Fig. 2, the reverse shock (or Mach disc) is Newtonian with $\gamma_{\mathrm{rs}} \sim 1.013$, in this lower density region at $t=160$. This is in fact, totally different from the equivalent $1 \mathrm{D}$ problem, as clearly seen in effective polytropic index in Fig. 2. The Mach disc compresses the beam matter, increasing the density and decelerating the flow to $\gamma \sim 18$ (in the equivalent 1D case the shocked jet beam should have $\gamma=5$ ). Furthermore, the 2D result shows a relatively larger region of the jet head that is formed by shocked beam matter (compare the 1D cuts in Fig. 2). The sound speed of the shocked beam material reaches $\sim 0.2$ with an effective polytropic index 1.64. Also, this shocked beam material tends to expand laterally with a speed $v_{\text {lateral }} \sim 0.1$. Similar to the lateral dynamics of the swept-up, shocked matter, again a rarefaction wave propagates towards the axis, which progresses very slowly, and eventually the shocked jet head ends up denser and colder. Since the shocked external medium (in the forward bow shock) has already cleared the immediate surroundings of the beam, the shocked beam matter will hardly form a backflow.

The remarkable feature of jet beam propagation in a lowdensity region is thus its stability. There is a very weak influence of the external medium on the jet, which allows the jet to transport energy over a long distance, and we followed this process over about 200 light-crossing times of the jet beam radius. Only a small fraction of the jet energy is transferred to the external medium in the inner region, through the bow shock. During the propagation in the inner region, there is no strong backflow and we hardly see the formation of a turbulent shear layer. In this lower region, the Lorentz factor of the jet beam and even the shocked jet beam, remains relativistic with $\gamma \sim 20$. In turn, the jet head propagates relativistically with a Lorentz factor 5. It must be clear from the above discussion that even in the lower density region, the inclusion of a realistic EOS is necessary as the forward shock is relativistic and the reverse shock (Mach disc) is Newtonian.

\subsubsection{Other models when traversing the lower medium}

In model $\mathrm{C}$, which is a light jet in this lower region at beam Lorentz factor 10, the jet mainly interacts with the external medium through the bow shock. Because of its lighter density, a turbulent cocoon gets formed during propagation. The turbulent cocoon also interacts laterally with the jet beam by compressing it, inducing internal shocks in the jet beam. The distance between shocks is about $\lambda_{\text {shock }}=20 R_{\mathrm{b}}$. At each shock the jet undergoes a small deceleration to Lorentz factor $\gamma \sim 9$ and is accelerated again to Lorentz factor $\gamma \sim 10$ due to the expansion of the jet after each shock. Thus, in this lower region the jet remains relativistic, its energy is mainly kinetic, and only a very weak fraction of the energy is transferred to the external medium by entrainment. The interaction of this light jet with the external medium also shows the formation of a low speed, higher pressure sheet of thickness $\sim R_{\mathrm{b}} / 2$ surrounding the inner jet.

Model $\mathrm{D}$ is similar to $\mathrm{C}$, but has the jet emerging with an initial open angle of $\theta=1^{\circ}$. In the first part, the $D$ jet undergoes a free expansion, and its density and pressure decrease until the pressure in the surrounding cocoon becomes high enough to compensate for the jet's thermal and ram pressure in the lateral direction. One can witness that an oblique shock forms in this region, which moves with a slow speed $v \sim 0.3$ that decreases during the jet propagation. In fact, as the jet propagates forward, the pressure of the cocoon behind the jet head decreases with cocoon expansion, and then the oblique shock moves forward. At $t=820$, the oblique shock is at the normalised distance $Z \sim 5$ (Fig. 11). This oblique shock in fact decelerates the jet to $\gamma_{\mathrm{b}} \sim 8$ and consecutively collimates it cylindrically, to end up with a smaller radius than the initial one imposed at inlet. Beyond the oblique shock, the jet beam is unstable and subject to the development of internal shocks. The amount of the matter entrained by the jet is greater than in the $\mathrm{C}$ case (cylindrical inlet). In this part of the jet, the internal energy increases and the state of the matter becomes mildly relativistic with an effective polytropic index falling to $\Gamma_{\text {eff }} \sim 1.55$. The jet in this model $D$ is then more stable than the jet in the model $\mathrm{C}$, since the initial conical propagation of the jet decreases the lateral interaction of the jet with the external medium and then the jet propagates in a laminar way until it reaches the collimation shock. 
Model E only differs from $\mathrm{C}$ in its twice higher beam Lorentz factor. It has an inertia ratio between jet-external medium of a similar order to model C. Despite the faster jet beam, its Lorentz factor along the beam in the first region ends up oscillating between 10-25 due to the successive internal shocks and jet expansions, such that the jets in both model show the same general behaviour. In the model $\mathrm{F}$, the $\mathrm{E}$ (cylindrical) jet model now emerges conically with an initial open angle of $\theta=1^{\circ}$. Its Lorentz factor $\gamma_{\mathrm{b}}=20$ and kinetic luminosity $L_{\mathrm{Jet}, \mathrm{Kin}}=$ $10^{43} \mathrm{ergs} / \mathrm{s}$ mean that the difference with the conical model D is a faster jet. In this model $\mathrm{F}$, the forward shock that forms at the jet head is stronger, producing higher pressure and a more extended cocoon. This cocoon limits the region of the free conical expansion of the jet by forming oblique shocks that recollimate the jet. This oblique shock in model F propagates with a speed $v \sim 0.1$, which is lower than the speed of the oblique shock in model D. As in model D, the speed of the oblique shock decreases during the propagation. Beyond this oblique shock, the Lorentz factor of the jet decreases to about 10, and the jet starts to be unstable with the formation of internal shocks that compress and induces consecutive acceleration and deceleration of the jet. In this region, the state of the matter in the jet is relativistic with an effective polytropic index $\Gamma_{\text {eff }} \sim 1.45$. At $t=820$, the oblique shock is at a normalised distance $Z \sim 7.5$ (Fig. 11).

In model $\mathrm{G}$, the interaction between the jet and the external medium is roughly the same as in model A, because only the Lorentz factor of the jet beam changes from $\gamma=20$ in model A to $\gamma=10$. In fact, the main differences between the two models is in the details of the structure of the jet head, since the rate of compression at the front shock and the Mach disc (reverse shock) is different. In any case, the jet interacts weakly laterally with the external medium and it remains stable, consistent with its higher density with respect to the surroundings. Finally, in the conical models $\mathrm{H}$ and I (otherwise similar to $\mathrm{G}$ and also of high energy), the jet only interacts with external medium through the shock in front, and there is no lateral interaction between the jet and the external medium, as only the head of the jet is slightly collimated. In fact, the conical propagation of the jet decreases the influence of the low-density external medium on the jet.

\subsubsection{Summary for the lower region}

In the lower region, the jet head propagates with a speed $v_{\text {head,Low }} \sim 0.6$ for the models $\mathrm{C}, \mathrm{D}, \mathrm{E}, \mathrm{F}$, and with a speed $v_{\text {head,Low }} s \sim 0.99$ in the models A, B, G, H, I, J, as also collected in Fig. 12. In fact, these two groups are clearly distinct from the (input) kinetic luminosity of the jet beam. The low-energy first group finds the lighter density jets interacting strongly with the external medium and slowing down, since the power of the jet beam in this group is $L_{\text {jet,Kin }} \sim 10^{43} \mathrm{ergs} / \mathrm{s}$, and then the inertia ratio between the jet and the external medium is low, and at most about $\eta_{\mathrm{R}} \sim 2.73$. For the second high-energy group, the external medium influences the jet weakly, since the power of the jet beam is $L_{\text {jet,Kin }} \sim 10^{46} \mathrm{ergs} / \mathrm{s}$ and then the inertia ratio between the jet and the external medium is high, up to about $\eta_{\mathrm{R}} \sim 3000$. Thus the variation in the Lorentz factor 10 to 20 of the jet beam and the small variation in the opening angle of the jet (cylindrical case versus conical with a small opening angle $1^{\circ}$ ) do not significantly influence the speed of propagation of the jet head in the lower scale region, which is simulated here up to 200 jet radii. While we have pointed out various aspects that are clearly captured only using a relatistic EOS model, our findings for jet propagation through the uniform, lower region are fully consistent with earlier works.

\subsection{Upper region propagation}

\subsubsection{Model A}

We now turn to the second stage in the dynamics, after the jet has passed the density jump. In model A, the density ratio between the jet and upper external medium suddenly changes to $\rho_{\mathrm{Up}} / \rho_{\mathrm{b}} \sim 4.687$, making it a light jet. In this denser upper region, the initial interaction between the jet and dense upper medium is shown in a zoomed Fig. 3 at time $t=240$. First, as the jet penetrates the denser medium, an oblique shock propagates laterally in the upper medium. The relatively higher density of the upper medium gives rise to reflection of this shocked matter, thus producing a somewhat more turbulent and hot cocoon. At the jet surface, a thin, rarefied, and very hot region develops (seen best in the low effective polytropic index in Fig. 3). In fact, the head of the jet now sweeps more matter up, enhancing the temperature. The high pressure in this region in turn gives rise to another oblique shock, which is weaker but propagates upstream and toward the axis. This confines the jet. This shock is located at time $t=240$ at about $Z \sim 9.8$. The jet radius drops due to this compression to about $R=0.03$.

Again we can learn from the analogous 1D Riemann problem and compare it with the axial structure of the $2 \mathrm{D}$ result. This is done in Fig. 4, at a time corresponding to Fig. 3. As a 1D shock-structured jet head penetrates in the high-density medium in an upper region, there are in fact four layers of shocked material that can be distinguished along the beam axis. Once the previously formed forward (bow) shock meets up with the density jump, a new forward shock develops that seperates swept-up high-density external medium from static external medium. A second contact or work surface from then on seperates shocked high-density matter from then previously sweptup, shocked, lower-density matter. From this same location, a new reverse shock forms that traverses the previously formed structured jet head (i.e. consisting of shocked lower matter and shocked beam matter and ending with the old Mach disc or reverse shock). These add up to 5 discontinuities in total, which are clearly seen in the analogous $1 \mathrm{D}$ problem shown in Fig. 4. However, the 2D jet propagation is different from this 1D model. The difference is quantified in Fig. 4, as we draw the cut along the $Z$ axis (middle row), and also at a fixed radial distance away from the axis at $R=0.02$ (bottom). The latter radius still remains in the jet "spine", since the laterally bounding shear region that we also can detect in our jet beam variation extends from 0.025 to 0.03 parsec.

We can clearly see that, when comparing these three cases, we find faster beam flows immediately behind the front shock in 2D, which are also relativistic with a Lorentz factor $\gamma \sim 4$. This is accompanied by strong compression, as the effective polytropic index drops to $\Gamma_{\text {eff }} \sim 1.37$. This is like the 1D case. To be precise, at time $t=240$, the front shock reaches a distance $Z \sim 12.75$ pc in the $2 \mathrm{D}$ case, where the $1 \mathrm{D}$ reaches $Z \sim 12.8$. Various internal shocks develop behind the front shock in the 2D case, partly induced by the initial structure of the jet beam with its interaction with the denser medium. At each new oblique shock, the flow undergoes a weak acceleration behind it. In the region of the shocked beam, the Lorentz factor along the axis oscillates between $\gamma_{\min } \sim 16$ and $\gamma_{\max } \sim 18$. Then, the trailing reverse shock (Mach disc) and other shocks in front of it are Newtonian along the axis, and the effective polytropic index oscillates between $\Gamma_{\text {eff,min }} \sim 1.65$ and $\Gamma_{\text {eff,max }} \sim 1.61$. In contrast, along the spine at fixed radius $R=0.02$, the reverse shock (Mach disc) and all shocks in front of it are stronger. In fact, the Mach disc is near-Newtonian (effective polytropic index $\Gamma_{\text {eff }} \sim 1.58$ ), 


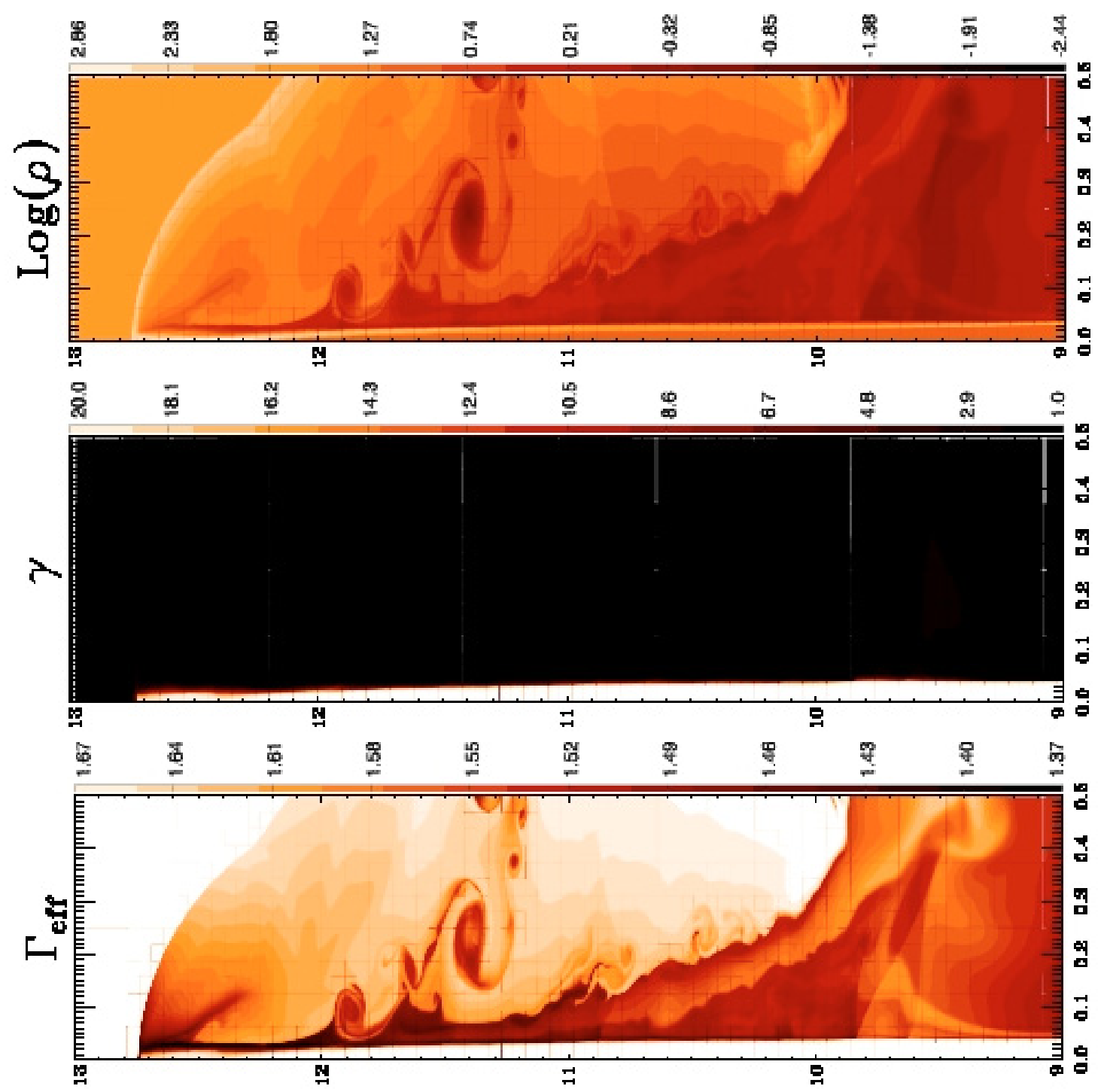

Fig. 3. A zoom on the jet head after penetrating the denser medium, for case A. We zoom with $R, Z \in[0,0.5] \times[9,13]$ parsec, at $t=240$. Top: logarithm of density, middle: Lorentz factor, bottom: effective polytropic index.

whereas the other shocks are relativistic with the effective polytropic index oscillating between $\gamma_{\min } \sim 1.34$ and $\gamma_{\max } \sim 1.46$.

There are thus clear $2 \mathrm{D}$ effects, such as the lateral compression of the jet by the hot cocoon and the presence of the oblique Mach disc. The latter at the same time shocks the jet beam, while compressing it laterally. A kind of reflection happens (see also Fig. 3), and the jet radius increases again in front of the reflection point, and gives rise to a weak acceleration. We find almost no backflow along the jet in this upper region. In fact, parallel to the jet, the hot shell of shocked external medium (as discussed above), moves in the same direction with a speed $v_{z} \sim 0.4$. The difference in speed between this shell and the surrounding slower and denser cocoon makes this region subject to instability, as seen clearly in Fig. 3. Meanwhile, the jet itself remains relatively stable. It seems that the formation of dense shear in the outer region of the jet increases the jet stability. However, in the jet, weak internal shocks are induced by the compression. The end result is the formation of a knot with long wavelength, and this result is shown in Fig. 5 where we plot the logarithm of the density and Lorentz factor at a later time $t=380$. We also show the variation in density and Lorentz factor along a larger section of the jet axis at this endtime, in Fig. 6. It can be seen that several internal shocks have developed, reminiscent of knots.

From this first model, it appears that a mildly overdense external medium cannot induce the strong deceleration observed in FR I jets, as the jet head for this case A remains relativistic in this region. However, this model could be relevant for FR II jets, as it reproduces the weak interaction with the external medium in the inner region, and stays stably structured in the upper region.

\subsubsection{Upper region: model B}

In model $\mathrm{B}$, the density ratio between the jet and the denser external medium is increased to $\rho_{\mathrm{Up}} / \rho_{\mathrm{b}}=671.220$. The interaction of the jet with this very dense external medium is very strong and entails the formation of a turbulent cocoon. In Fig. 7, we show the density in the entire simulation region at our endtime $t=900$. In this model, the inertia ratio between jet and external medium was small $\eta \sim 0.0604$, making the jet beam subject to instabilities. A zoom of the jet head at this same endtime is also shown in the last panel from Fig. 8. 

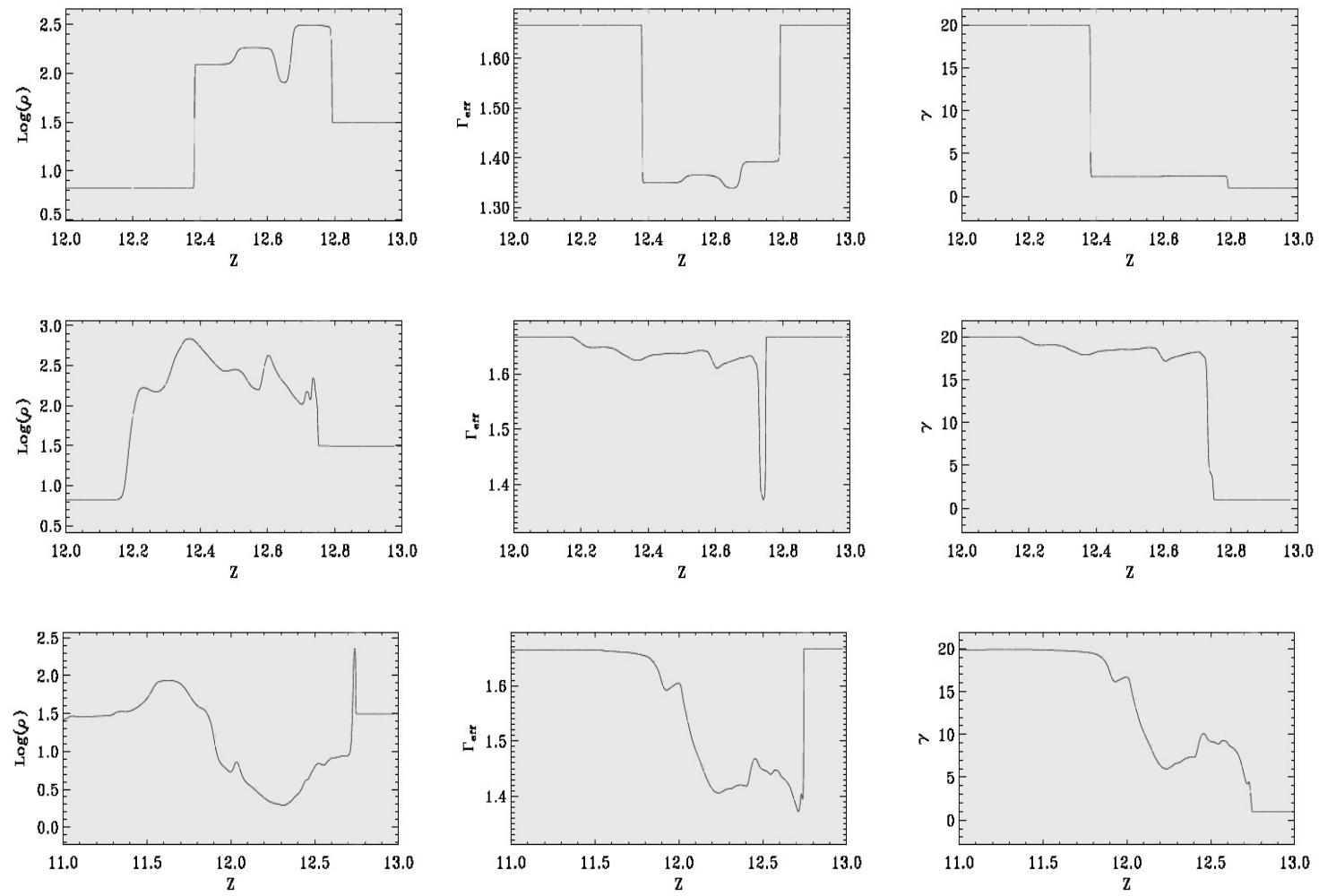

Fig. 4. Case A in the upper medium. Left: logarithm of density, centre: effective polytropic index. Right: the Lorentz factor, at time $t=240$, i.e. after penetrating the denser medium. The three rows correspond to: (top) a $1 \mathrm{D}$ equivalent relativistic shock problem, (middle) a cut along the $Z$ axis (i.e. $R=0$ ) through the jet head, and (bottom) a cut along the axis at a radius $R=0.02$. From the top to the bottom, note the difference in scale: $Z \in[12.0,13.0]$ for top and middle, while bottom panel has $Z \in[11.0,13.0]$.

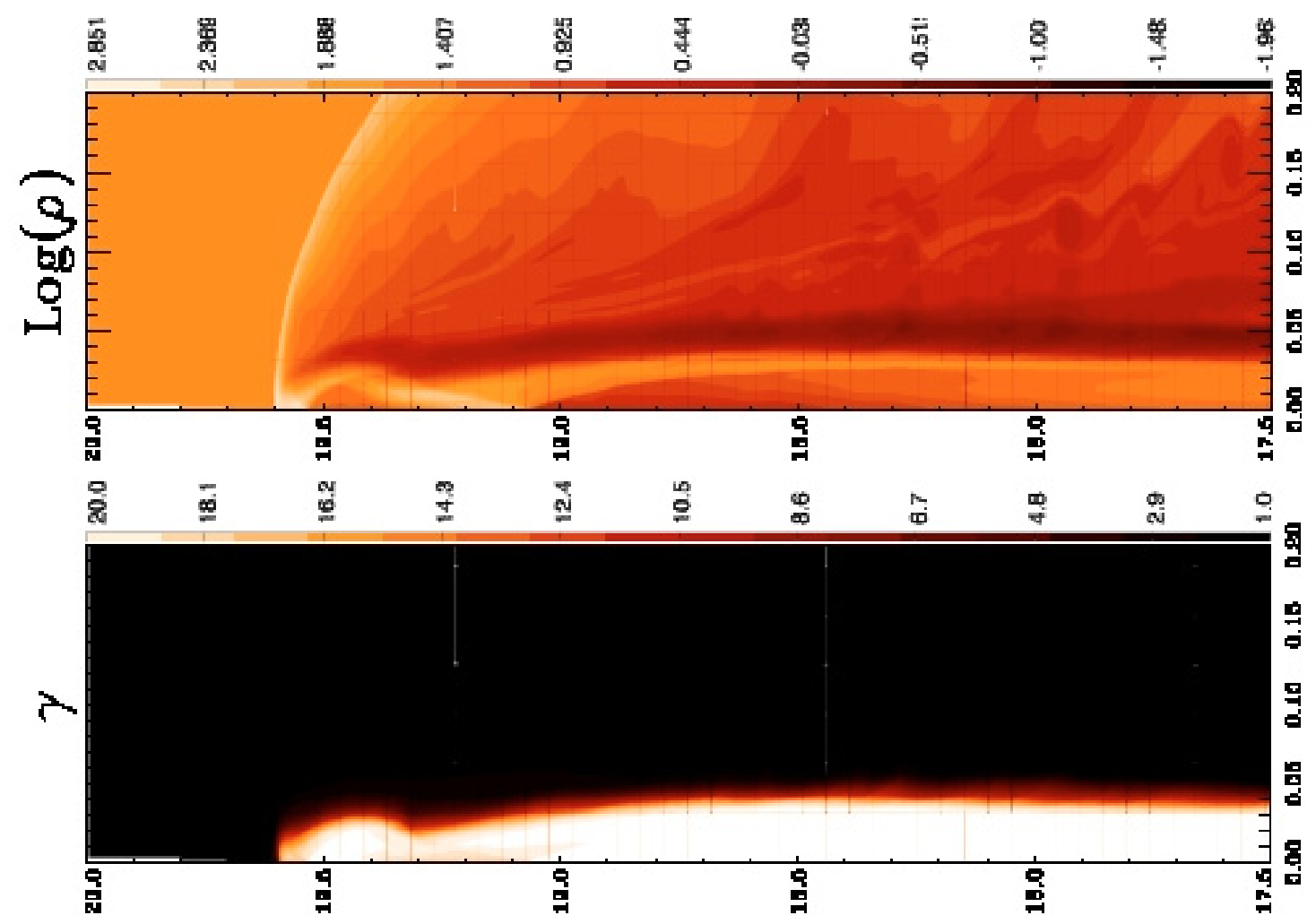

Fig. 5. A zoom on the jet head with $R, Z \in[0,0.2] \times[17.5,20]$, at $t=380$, for case A. (Top) Logarithm of density, (middle) Lorentz factor, (bottom) effective polytropic index. 

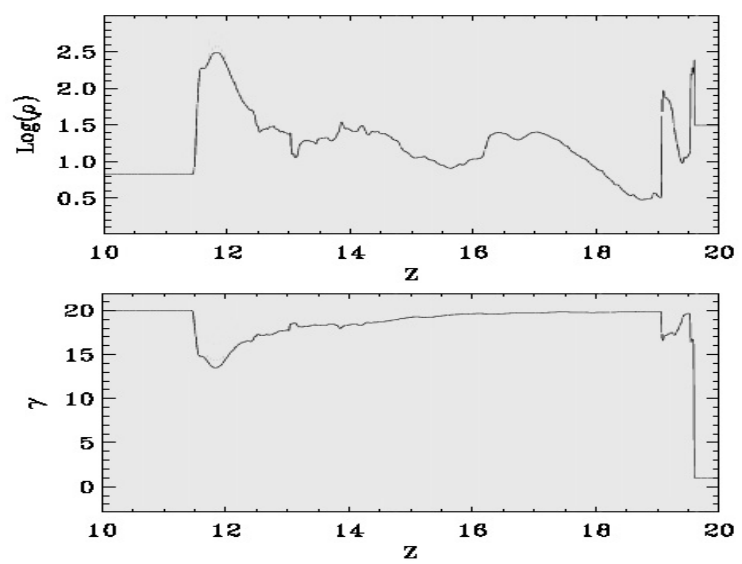

Fig. 6. A cut through the jet for case A along the $Z$ axis, at $t=380$. Top: the logarithm of density, bottom: the Lorentz factor.

The result of the interaction is the formation of a bow shock propagating in front of the jet beam and to the side. The shocked external medium initially spreads laterally with a speed $v_{\text {lateral }} \sim$ 0.18 , lower than the sound speed. The high density of the external medium leads to a weak compression rate, and in fact, the front (forward) shock is now Newtonian with an effective polytropic index $\Gamma_{\text {eff }} \sim 1.6$. The compression rate is about 5 and the jet head propagates with a Lorentz factor less than 1.25 along the axis and more slowly away from the axis. Indeed, along the line $R=0.01$, the shock propagates with a Lorentz factor 1.09 (Fig. 9).

The slow spreading of shocked dense matter makes the $2 \mathrm{D}$ effects on the propagation of the forward shock less significant than in the first model, and there is less difference with a similar 1D case. Again, Fig. 9 compares analogous 1D results with axial cuts along axis and at $R=0.01$, for an earlier time $t=240$ (for which 2D impressions are shown in the top two panels of Fig. 8). The shocked jet beam in 2D is structured axially as in the 1D case with the appearance of a new work surface and the Mach disc, while the compression rate at the trailing Mach disc approaches the 1D case. However, we see that the effective polytropic index there is higher, namely $\Gamma_{\text {eff }} \sim 1.4$, and the Lorentz factor is different $(\gamma \sim 1.03$ in the $1 \mathrm{D}$ and $\gamma \sim 6$ in the $2 \mathrm{D}$ jet). The resemblance of the structure of the shocked external medium to the jet beam in $1 \mathrm{D}$ versus $2 \mathrm{D}$ is better near the jet axis. We can clearly detect the new forward shock and the second contact discontinuity, seperating the shocked highdensity matter with previously swept-up matter from the lower density region. The differences between $2 \mathrm{D}$ and $1 \mathrm{D}$ are the additional oscillations on the density and pressure, which are due to the lateral effects. Sideways spreading matter is strongly reflected, as the high density of the external medium slows down the lateral growth of the cocoon. Moreover, the oblique Mach disc confines the jet. Beyond it, the jet radius increases and the flow is again accelerated to reach a Lorentz factor $\gamma \sim 17$. Then, the hot and dense cocoon compresses the jet again, and a new internal shock decelerates the jet. Along the line $R=0.01$, the reverse shock is Newtonian and there is evidence of more internal shock development, indicating complex interaction with the cocoon.

In this case B, we do find strong backflows. The shocked jet beam matter by the trailing Mach disc again is subject to sideways spreading. The denser hot cocoon reflects this spreading matter and induces the formation of a backflow. In fact, between the jet and the shocked external medium, a shear layer develops,

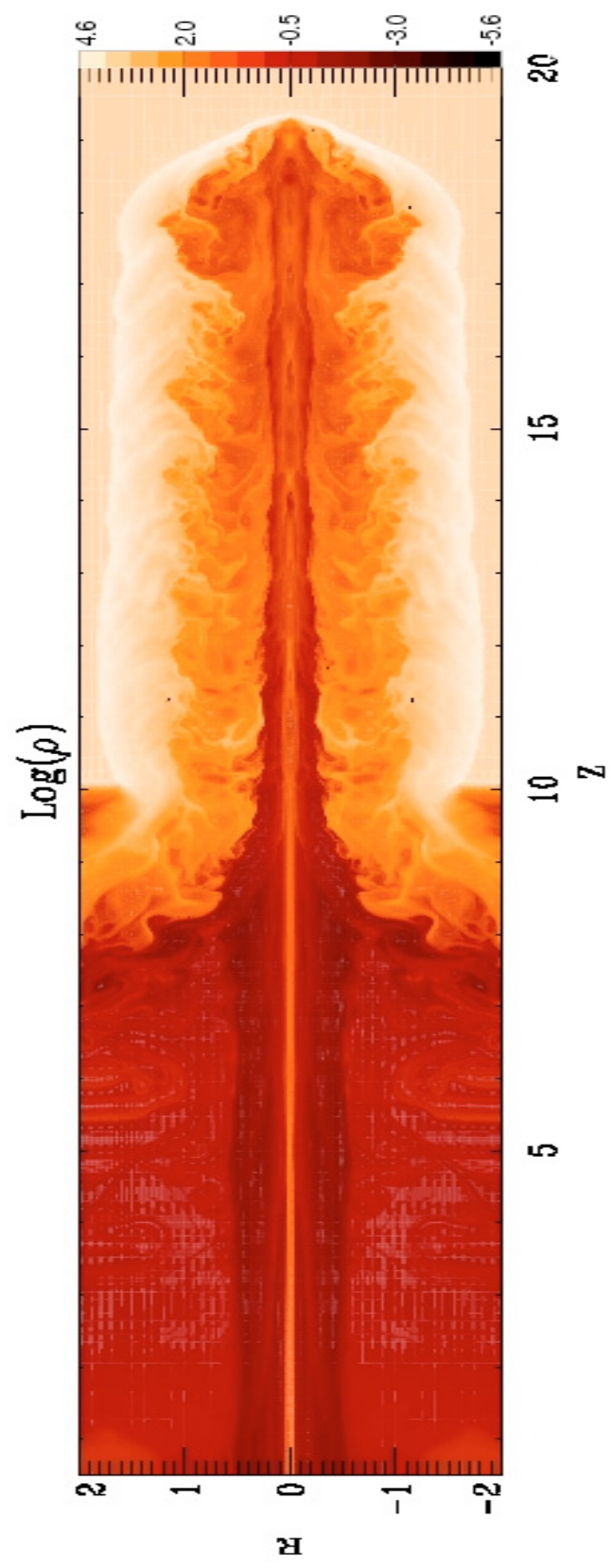

Fig. 7. The logarithm of density at $t=900$ on the entire simulation domain, for the high-density, upper medium case B.

consisting of a dense and relatively hot flow propagating upstream with a speed of $v_{\text {backflow }} \sim-0.2$. Near the top of the jet head, the backflow essentially consists of shocked jet beam matter, flowing in a cylindrical shell of thickness $\Delta R \sim 0.01$ parsec. The mass flux in this backflow increases in the upstream direction. Moreover, this backflow entrains shocked external cocoon matter with it, and then carries more mass, which slows down its speed to $v_{\text {backflow }} \sim-0.1$ when it reacheas $Z=10.0$ (the original position of the density jump in the external medium). The backflow also interacts strongly with the non-shocked jet beam, and it compresses the jet beam and induces shocks. The final result of this fairly complex interaction is confinement and overall deceleration of the jet in the upper region. The jet radius decreases to $R \sim 0.02$ in $Z \sim 5.0$, at $t \sim 900.0$. Beyond this point the jet radii start to increase. And a strong oblique shock forms at the interface between the two medium. The jet is strongly decelerated 

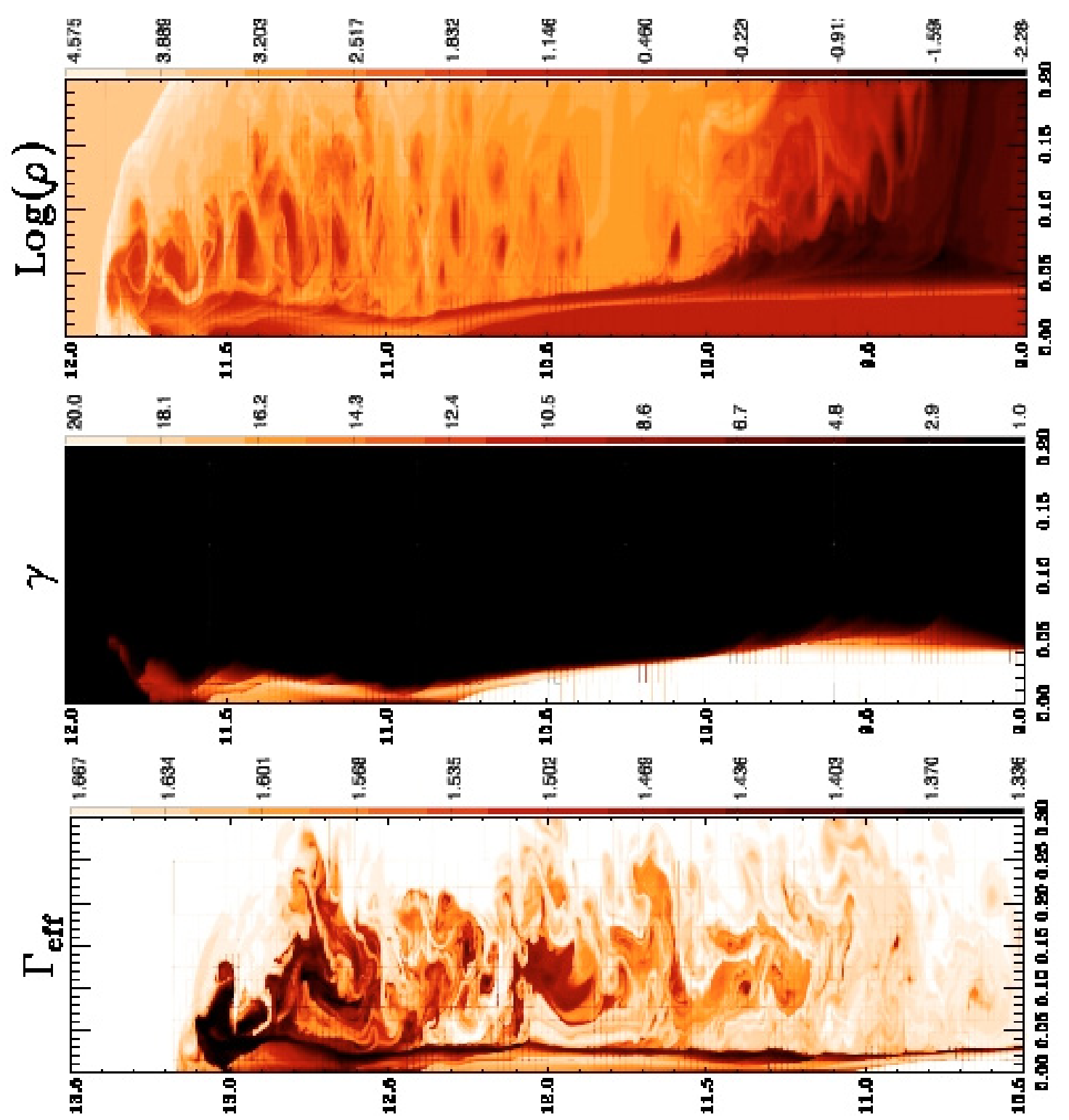

Fig. 8. A zoom on the jet head after penetrating the high-density medium in case $\mathrm{B}$, for $R, Z \in[0,0.2] \times[9,12]$ top and middle panel show at $t=240$ : (top) Logarithm of density, (middle) Lorentz factor. Bottom panel shows, later at $t=300$, the effective polytropic index, zoomed with $R, Z \in[0,0.3] \times[10.5,13.5]$.

at this shock to $\gamma \sim 2.0$ and the pressure increases, such that the relativistic mach number falls to $M \sim 5$. The high-pressure jet expand laterally in this region to a radius $R \sim 6 \times R_{\text {jet, } \mathrm{b}}$.

The interaction of the backflow with the shocked external medium in the cocoon induces the development of Kelvin-Helmholtz instabilities. This is the result of the velocity gradient between the backflow and the cocoon, and also occurs because the backflow is slightly underdense with respect to the cocoon. It is clear from Fig. 8 that the entire region is highly structured due to instability development. In this upper region, the density ratio was $\rho_{\mathrm{Up}} / \rho_{\mathrm{b}}=671.220$, and we indeed find a transition to a beam suddenly traveling at a Lorentz factor of $\gamma_{\mathrm{Up}} \sim 1.25$. This value is a bit higher than the value used for setting up the initial condition (where we used 1.02). This difference can be explained, by the head of the jet beam being made up of hot swept-up matter and hot shocked beam matter, while the estimate assumed cold conditions. Moreover, in this upper region, the forward bow shock is now Newtonian. The various layers at the head of the jet give rise to strong turbulence development. A result of this interaction and the entrainment of the externa matter by the jet is the deceleration of the jet in the upper region to $\gamma \sim 1.5(v \sim 0.3)$.

When estimating the overall energy budget, we find that in the jet interaction with the upper medium, about $58 \%$ of the jet energy gets deposited in the upper lobe, while a fraction around $10 \%$ is reflected in the lower region.

\subsubsection{Other cases: effects of opening angle and density decrease}

Low-energy jets. In model $\mathrm{C}$, the density ratio between the jet and the dense external medium is $\rho_{\mathrm{b}} / \rho_{\mathrm{Up}}=4.91 \times 10^{-3}$. There is strong interaction of this light jet with the external medium, and this implies the formation of a high-pressure cocoon. This turbulent cocoon disturbs the jet by inducing a strong backflow. The difference in pressure between turbulent cocoon and the colder lower region also produces backflows propagating into the lower region. At the interface between the two regions, the jet beam is 

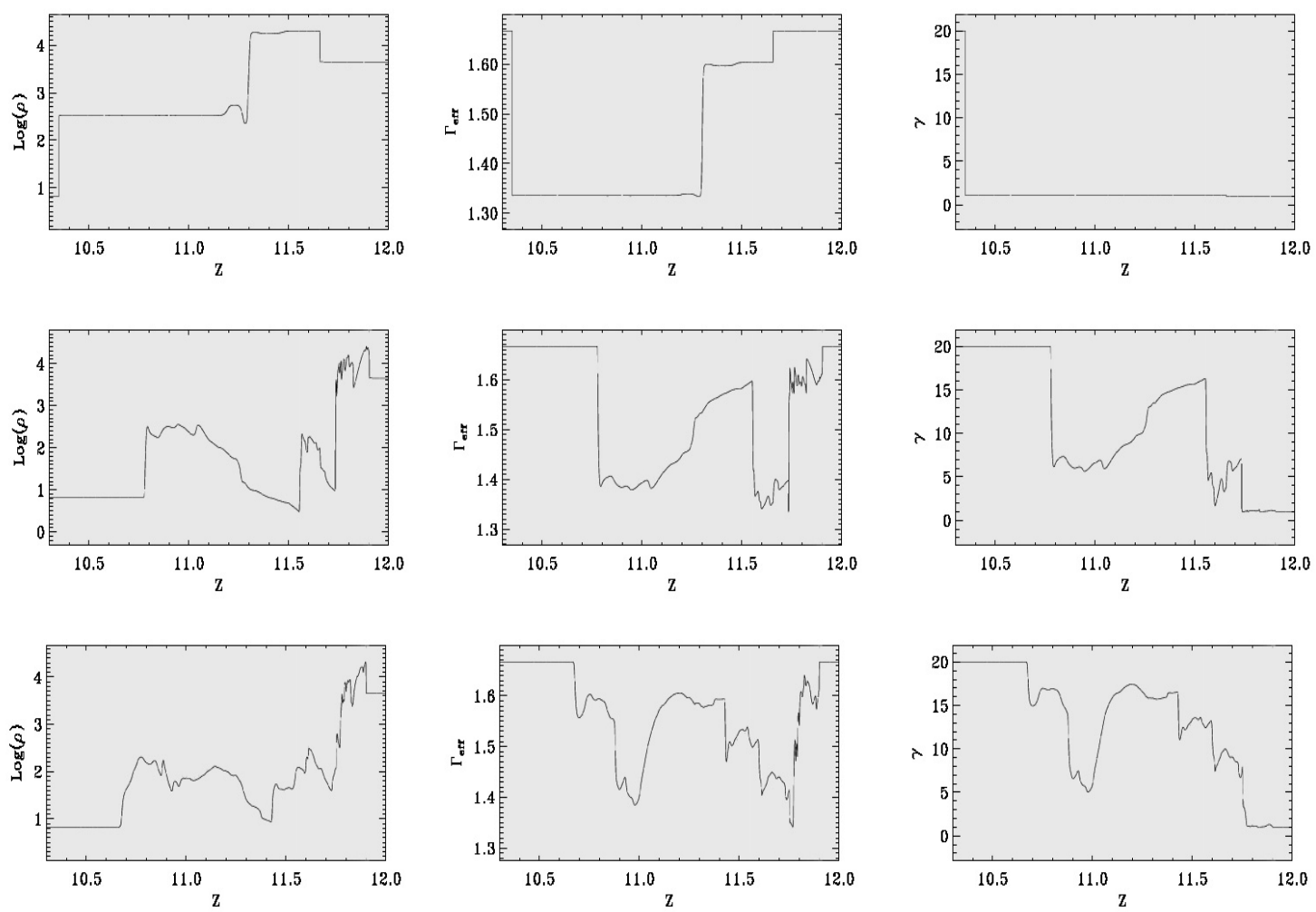

Fig. 9. (Left) logarithm of density, (centre) effective polytropic index, (right) Lorentz factor, for three cases. The three rows are (top) a 1D equivalent relativistic shock problem, (middle) a cut along the $Z$ axis on the case B jet head at $t=240$, (bottom) a cut along the axis at fixed $R=0.01$.

compressed. The jet becomes unstable and its pressure increases. However, the jet remains relativistic in the upper region, since it only decelerates to a Lorentz factor $\gamma \sim 8$. The decrease in the density in the external medium as assumed for this jet (see Table 1) and the (minor) deceleration of the jet makes the forward shock weaker, and then the pressure of the cocoon at the head of the jet decreases. Therefore, the jet beam radius actually increases slowly during propagation in this upper stratified region.

In model $\mathrm{D}$, the jet beam that finally emerges from the lower region is collimated cylindrically (despite its original opening angle, see the discussion in the previous paragraphs) and ends up with a relativistic Mach number $M \sim 20$ and with a Lorentz factor of $\gamma \sim 8$. This jet again interacts strongly with the external, upper medium forming a high-pressure cocoon that induces a backflow, also propagating upstream in the low density region. This backflow compresses the jet near the (perturbed) interface, increasing its density and pressure. The jet in the upper region is found to decelerate to a Lorentz factor $\gamma \sim 5$ through multiple internal shocks. As in model C, the compression of the jet at the interface increases its pressure, and the assumed density decrease in the external medium causes the jet to undergo a slight conical expansion in this upper medium.

In model $\mathrm{E}$ (main difference with $\mathrm{C}$ is that the jet is faster), we find an even stronger interaction with the external medium. This configuration enhances the development of the shear instabilities, which causes entrainment of ambient material. Then this jet $\mathrm{E}$ is found to decelerate smoothly to a Lorentz factor $\gamma \sim 5$, while it was characterised by $\gamma \sim 20$ in the inlet.

In model $\mathrm{F}$, the jet beam emerges from the upstream, lower region with a fairly low mach number $M \sim 15$ (lower than in model D) and with an internal energy close to the mass energy. Moreover, the jet in $\mathrm{F}$ propagates with a high Lorentz factor and higher thermal energy. When it interacts with the downstream, denser medium, it forms a flattened (compressed) and turbulent cocoon. Then a stronger backflow develops than in case D, which propagates upstream in the low-density region. This backflow this time eventually induces a deceleration of the jet to a Lorentz factor $\gamma \sim 10$ (along the axis). At the interface, the jet pressure ends up higher than the pressure of the cocoon, and this jet also expands in the upper region in a conical shape until it reaches pressure equilibrium with the cocoon in this region. The jet interacts strongly laterally with the cocoon in this region, and overall, the jet beam slows to a Lorentz factor $\gamma \sim 5$ (a long the axis) through multiple internal shocks induced by instabilities and entrainment of external matter.

High-energy jets. In model $\mathrm{G}$, the difference with model A is that the jet is slower and the upper medium less dense. The interaction of the $G$ jet with the external medium is therefore weaker than in A, and the jet also remains relativistic. At the end of simulation, this jet deposited $2.5 \%$ of its energy in the upper medium and a fraction of the order of $1.9 \%$ is reflected in the lower region. In model $\mathrm{H}$, which already had a conical expansion of the jet in the lower region, and when the jet reaches the interface between the two regions, the density of the external medium is 155.5 higher than the density of the jet. When the jet goes into the upper medium, a strong bow shock forms and a high-pressure cocoon develops. We also find a backflow that reaches a speed around $v_{\text {backflow }} \sim-0.5$ at the location of the interface. A shock wave forms there and propagates upstream in the lower density region. This shock wave compresses the 


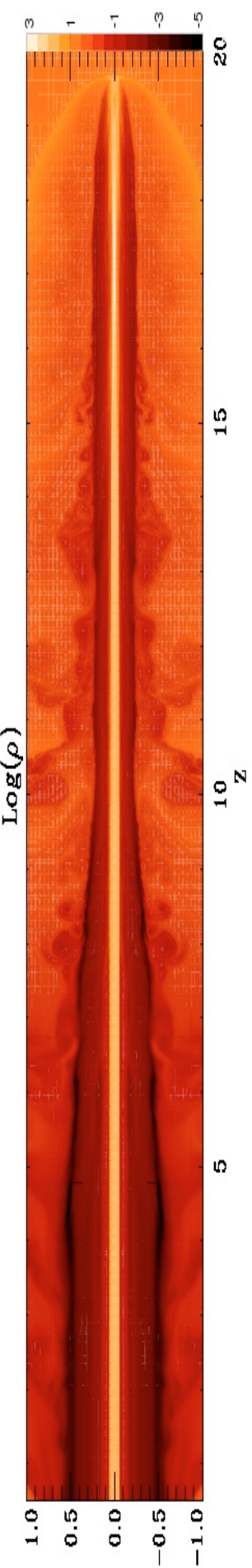

d

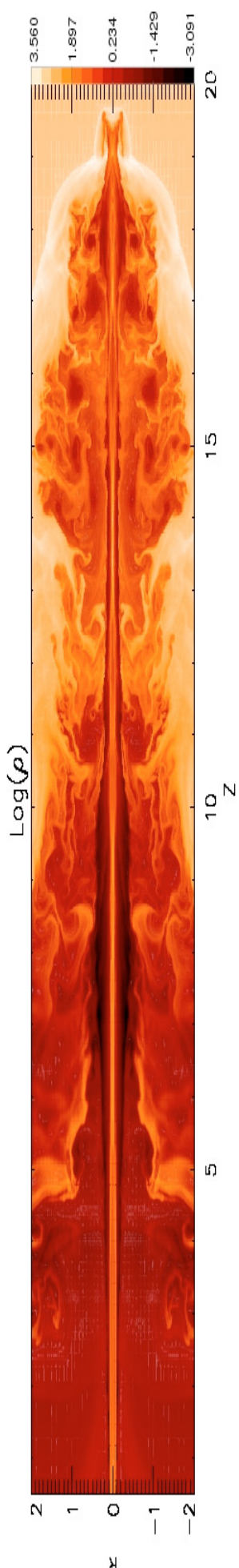

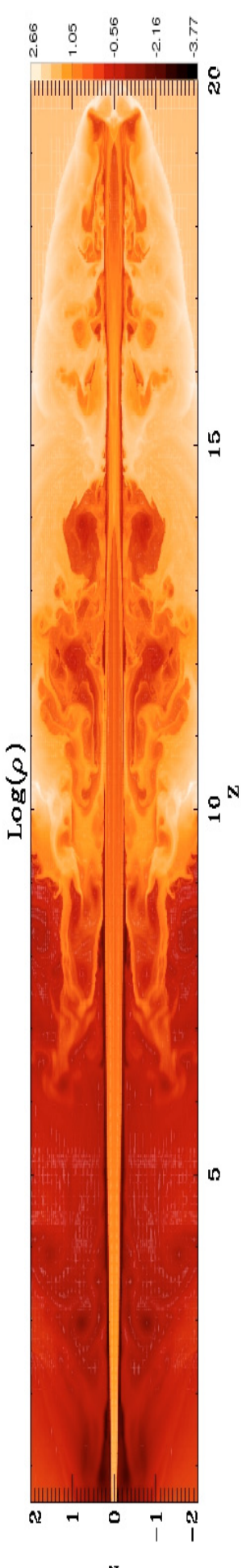

g

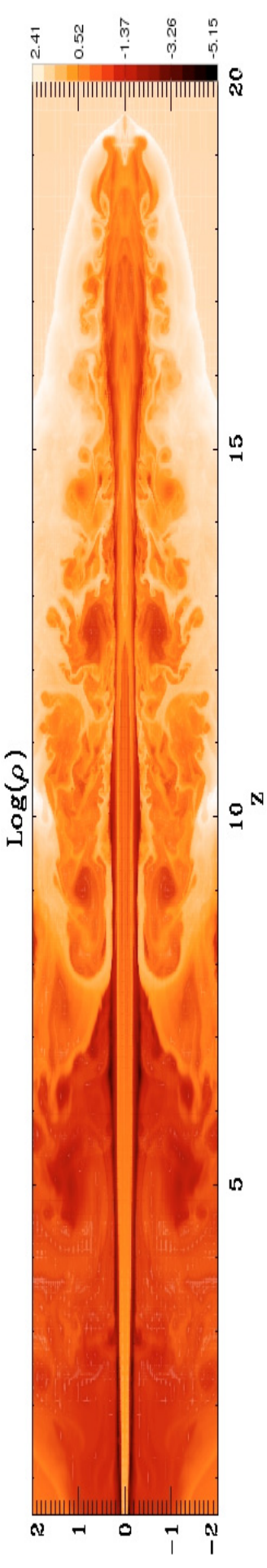

H

Fig. 10. Contours of logarithm of density for high-energy simulations: $\mathrm{G}$ at $t=380, \mathrm{H}$ at $t=800, \mathrm{I}$ at $t=480, \mathrm{~J}$ at $t=480 . R$ and $Z$ are normalised to $\left(20 \times R_{\mathrm{b}}\right)$.

jet beam and collimates it cylindrically near the interface between the two media. At $t=820$, the this shock wave reaches the normalise distance $Z \sim 5.0$ (Fig. 10). In the upper medium, the high-pressure cocoon induces the formation of an oblique shock at the jet head. This compresses and the jet radius changes from $R \sim 3.0 \times R_{\text {jet, }}$ to $R \sim R_{\text {jet, }} / 2.0$. In all, the jet decelerates abruptly from Lorentz factor $\gamma \sim 10$ to $\gamma \sim 2$ at this location. At this shock, the pressure becomes higher than the pressure in the cocoon, the state of the matter becomes relativistic and its effective polytropic index falls to $\Gamma \sim 1.34$. Beyond this shock, the jet beam spreads with an angle of about $\theta \sim 6^{\circ}$ (mostly influenced by the decreasing density) and is accelerated to a Lorentz factor $\gamma \sim 8$ before it reaches the bow shock. At $t=820$, the oblique shock is at the normalised distance of $Z \sim 11.0$ (Fig. 10). At the 
end of simulation, the $\mathrm{H}$ jet deposited $40 \%$ of its energy in the upper lobe and a $16 \%$ fraction is reflected in the lower region.

In model I, the difference with the model $\mathrm{H}$ is that the upper medium is less dense than the jet. In the initial period, the jet interacts with the external medium mainly through the front shock until the bow shock starts to develop prominently in the upper region and its pressure increases. The high pressure of the cocoon compresses the jet and induces an internal shock in the jet that collimates it. Beyond this shock, the jet becomes unstable and multiple shocks develop, decelerating the jet to Lorentz factor $\gamma \sim 8$. At $t=480$, the oblique shock is at the normalised distance $Z \sim 14.0$ (Fig. 10). At the end of simulation, this I jet deposited $18 \%$ of its energy in the upper lobe and a fraction about $8 \%$ is reflected in the lower region.

In model $\mathrm{J}$, the jet is faster than model $\mathrm{H}$ (Lorentz factor 20 on inlet) and the external upper medium has a density 2.5 lower than in model $\mathrm{H}$. The initial phase of the interaction of the jet with the upper external medium also produces a shock wave propagating upstream, collimating the jet cylindrically. Similar to model $\mathrm{H}$, the high-pressure cocoon that develops in the upper region causes a backflow that propagates upstream. This disturbs the jet there and induces the formation of an oblique shock that compresses, collimates, and decelerates the jet. In fact, at the shock, the jet radius now falls from $R \sim 4.0 \times R_{\text {jet,b }}$ to $R \sim R_{\text {jet, } \mathrm{b}}$. The jet is decelerated to a Lorentz factor $\gamma \sim 10$ through the shock. Beyond this, the jet spreads again and undergoes multiple internal shocks. Ultimately, this decelerates the jet beam to the Lorentz factor $\gamma \sim 5$. At $t=480$, the oblique shock is at the normalised distance $Z \sim 12.5$ (Fig. 10). The faster jet J propagates for a longer distance than the jet in $\mathrm{H}$. In fact, jet $\mathrm{J}$ traverses a longer distance in free ballistical propagation since the ram pressure in the jet beam is higher. Then the oblique shock forms farther away than in $\mathrm{H}$, and its overall compression rate is lower. At the end of simulation, the jet deposited about $18 \%$ of its energy in the upper lobe and a fraction $12 \%$ gets reflected.

\subsubsection{Summary of all cases}

Figures 10, 11 give an impression of the jet morphologies at the end of the simulations. In our models $A$ and $G$ with high energy and an upper medium of about the jet density, we end up with a relativistic jet in both regions (inner/upper). In the upper, slightly overdense region (for A), the interaction with the external medium is stronger than in the underdense inner region, and we find the formation of a relatively smooth cocoon. In model $\mathrm{B}$ with high energy and a much higher density in the upper medium, the jet is relativistic in the lower region, where it is seen to propagate with a Lorentz factor of about 5 , and turns to a sub-relativistic jet with propagation speed 0.3 in the very dense upper region. In all models with low energy $\mathrm{C}, \mathrm{E}$, the jet remains relativistic in the lower region despite fairly turbulent cocoons, and is strongly decelerated when it crosses the interface between the two media. In the cases undergoing strong deceleration, a shock wave propagates upstream (a reflection from hitting the higher-density environment up), and this compresses and decelerates the jet. In the model with an initial opening angle, an oblique shock forms before the jet reaches the contact interface between the two media for the cases D, F with low-energy. This shock survives the interaction with with dense external medium and gets enhanced by the shock wave propagating upstream that forms when the jet interacts with the upper medium. In conical models H, I, J with higher energy, such an oblique shock forms only when the jet starts to interact with the higher density upper medium. These pronounced differences in the propagation behaviour between the two jets are clearly seen when we quantify the propagation of the jet head, which is collected as a function of time in Fig. 12 for all models. We can conclude that this is relevant to the FR I/FR II dichotomy, because we find FR II behaviour where the energy of the jet is high and the density of the external medium is not high enough to significantly impart a strong reflected shock, and FR I behaviour when it can, even if the density beyond the interface decreases and even if the jet is not collimated.

Finally, in all these simulation, when the jet crosses the intersection between the two medium, we witness the appearance of Richtmyer-Meshkov instabilities at the shocked contact interface. We were able to resolve the local vortical development by using high resolution. In fact, the Richtmyer-Meshkov instabilities evolve out of vorticity deposition on the interface, which is due to the propagating jet bow shock passing it.

\section{Conclusions}

In this paper, we extended, presented, and applied the relativistic hydrodynamics AMRVAC code (Meliani et al. 2007; van der Holst \& Keppens 2007) with a generalised variable polytropic index equation of state for the purpose of modelling the relativistic shocks in GRBs and AGN jets. As follows in the Appendix, we used various shock-capturing schemes on a variety of test cases for adiabatic and non-adiabatic cases for code validation. We demonstrated the code ability with stringent new test problems, developed according to the astrophysical context. We also tested the code for a case with a heated flow, using the Synge-like equation of state from Meliani et al. (2004). The Riemann problems were also solved exactly, and the AMR simulations handled cases with Lorentz factors around 100 accurately.

We explored a new scenario for the sudden deceleration of relativistic jets in the FR I radio galaxies. This model for jet deceleration is based on a density jump in the external medium, with density suddenly increasing to the upper medium. We included models of conical jets with an initial opening angle and allowed for decreasing denisty profiles within the upper medium. We investigated the propagation and the dynamics of relativistic AGN jets over a long distance, resolving small-scale instabilities that develop in the cocoon and that could be responsible for particle acceleration. This study was only possible with adaptive mesh refinement. We quantified and discussed the deceleration of the jet resulting from its interaction with a layered medium. We point out that jet deceleration can be significantly aided by an internal oblique shock that forms in jets with conical expansion. Under fairly extreme (but not as extreme as pursued in other simulations to date) density conditions in the dense upper region, it can reproduce the strong deceleration observed in FR I jets.

In the early phase, the head of the cold, fast (beam Lorentz factor 10 or 20) jet propagates in the low-density medium at a high Lorentz factor $\gamma=5$. In cases with high energy, almost no cocoon or backflows develop in this phase, and as the jet is heavier than the external region, it behaves more ballistically. When observed in this region, the relativistic beaming is high, so that one would observe a one-sided jet. In low energy jets, a cocoon always forms and slow backflows develop. They in turn induce multiple internal shocks making the jet decelerate and re-accelerate. In jets with low-energy and with an initial conical flow, the flow remains ballistic until it reaches a self-consistently forming an oblique shock. This compresses and decelerates the jet, and two regions appear in the jet beam, the first with high 


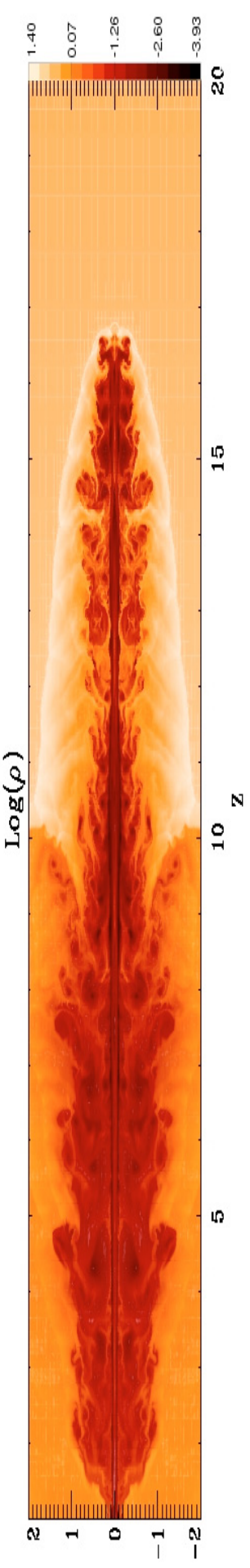

y

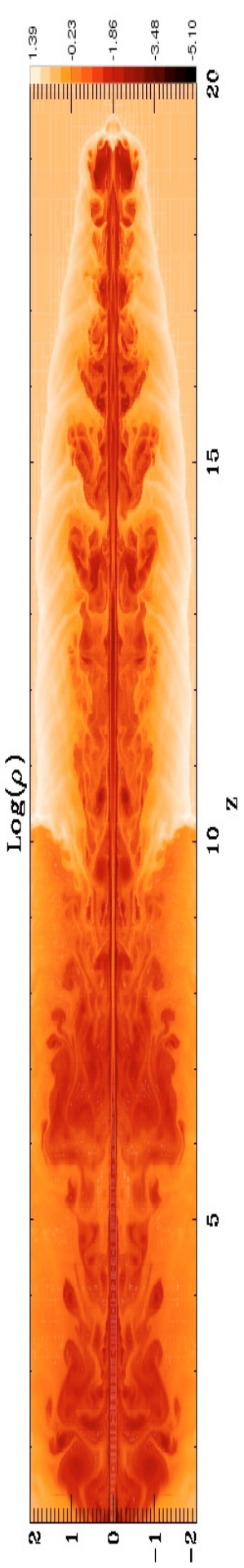

y

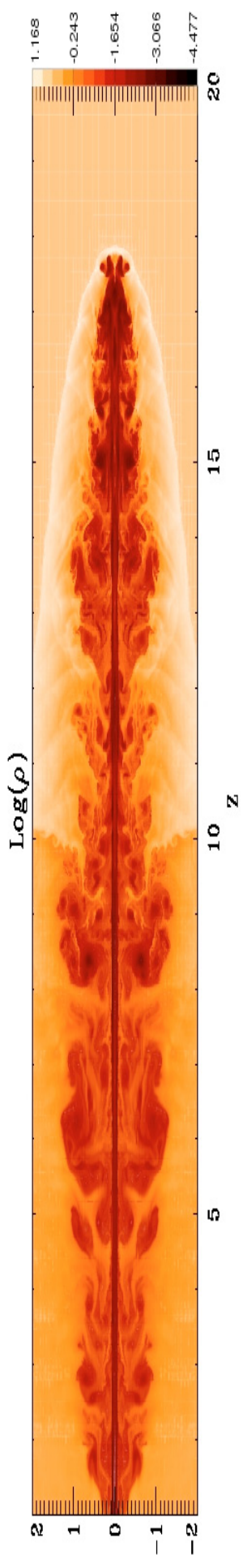

y

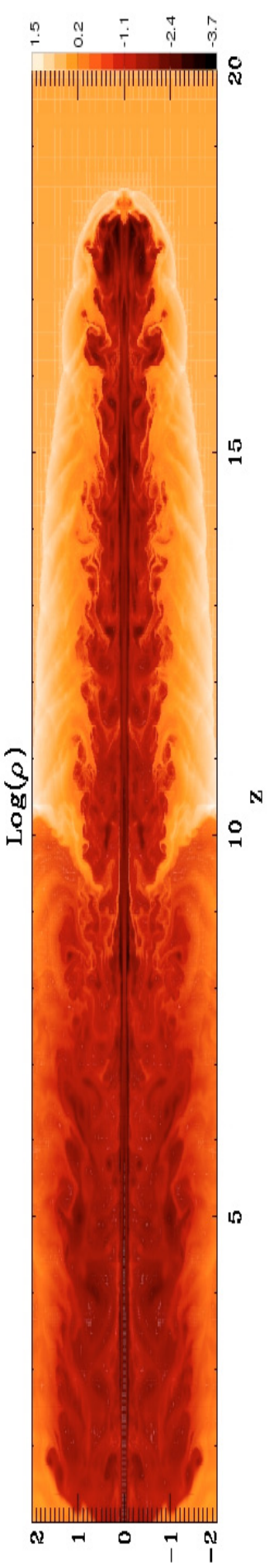

马

Fig. 11. A contour of the logarithm of density for low-energy simulations: $\mathrm{C}$ at $t=820, \mathrm{D}$ at $t=820, \mathrm{E}$ at $t=820, \mathrm{~F}$ at $t=820 . R$ and $Z$ are normalised to $\left(20 \times R_{\mathrm{b}}\right)$.

relativistic beaming and the second with low Lorentz factor, showing clear disturbances by the surrounding cocoon.

In the outer region where the density increases suddenly, we find increased entrainment of ambient material through velocity shear instabilities at the jet boundary and working surfaces. Here for the first time we address how the prior interaction of the jet with the low-density ambient medium in the inner region (which makes the head of the jet consist of swept-up ambient medium and a shocked beam) affects jet propagation in denser media. The pre-structured jet head has a lower Mach number, which gives rise to a strong interaction with the denser ambient medium.

For models with a weak increase in density in the external medium, the cocoon shows little turbulent structure and no backflow appears. The jet in this case remains stable and relativistic in 


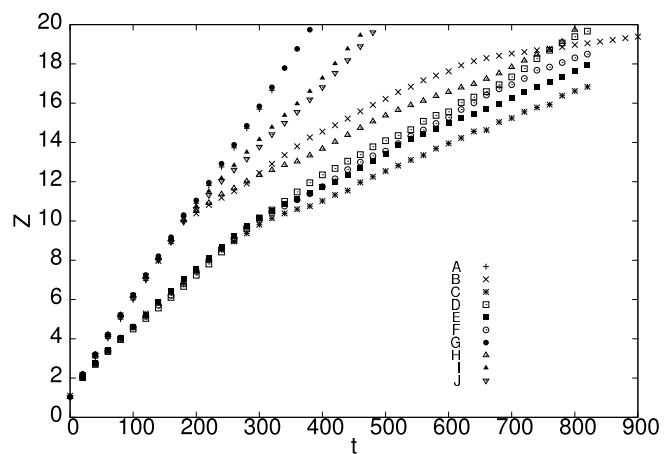

Fig. 12. The position of the jet head as a function of time, for all cases.

the denser upper region. Also a fraction of its energy is deposited in the external medium, but only through the frontal shock. This model could be relevant for jets in FR II. With this scenario, one can explain how most of the energy of the jet is deposited in the outer region, since relatively little jet energy gets transferred to the medium in the inner region.

Those models with more extreme increases in density in the external medium showed the formation of an overpressured and turbulent cocoon. A strong backflow develops in the outer region, which disturbs the jet structure. The backflow compresses the jet and induces internal shocks, which give rise to knot formation. The jet becomes subrelativistic within the simulated domain. The models with initial conical jets show the development of an oblique shock that decelerates the jet. In future work, we will follow these jets over increasingly larger distances in $3 \mathrm{D}$ scenarios. We also intend to provide synthetic observational radio maps from our computed jet models.

Acknowledgements. We acknowledge financial support from the Netherlands Organization for Scientific Research, NWO-E grant 614.000.421, and from the FWO, grant G.027708, and computing resources supported by NCF. Part of the computations made use of the VIC cluster at K.U. Leuven.

\section{References}

Allen, S. W., Dunn, R. J. H., Fabian, A. C., Taylor, G. B., \& Reynolds, C. S. 2006, MNRAS, 372, 21

Aloy, M. A., Ibáñez, J. M., Martí, J. M., \& Müller, E. 1999, ApJS, 122, 151

Blandford, R. D., \& McKee, D. G. 1976, Phys. Fluids, 19, 1130

Bicknell, V., \& Begelman, M. C. 1996, ApJ, 467, 597

Bridle, A. H. 1992, Testing the AGN paradigm, AIP Conf. Proc., 254, 386

Canvin, J. R., Laing, R. A., Bridle, A. H., \& Cotton, W. D. 2005, MNRAS, 363, 1223

Carilli, C. L., \& Barthel, P. D. 1996, A\&ARv, 7, 1

Choi, E., Wiita, P. J., \& Ryu, D. 2007, ApJ, 655, 769

Cohen, M. H., Lister, M. L., Homan, D. C., et al. 2007, ApJ, 658, 232

Croft, S., van Breugel, W., de Vries, W., et al. 2006, ApJ, 647, 1040

Daly, R. A. 1995, ApJ, 454, 580

Del Zanna, L., \& Bucciantini, N. 2002, A\&A, 390, 1177

De Young, D. S. 1993, ApJ, 405, L13

De Young, D. S. 1996, in Energy Transport in Radio Galaxies and Quasars, ed. P. E. Hardee, A. H. Bridle, \& J. A. Zensus, ASP Conf. Ser., 100, 261

Drake, C. L., McGregor, P. J., Bicknell, G. V., \& Dopita, M. A. 2003, PASA, 20, 57

Duncan, C., \& Hughes, P. 1994, ApJ, 436, L119

Duncan, C., Hughes, P., \& Opperman, J. 1996, ASPC, 100, 143

Einfeldt, B. 1988, on Godunov-type methods for gas dynamics, SIAM J. Numer. Anal., 25, 294

Eulderink, F., \& Mellema, G. 1994, A\&A, 284, 654

Evans, D., Lee, J., Fong, W., et al. 2008, ApJ, 675, 1057

Fanaroff, B. L., \& Riley, J. M. 1974, MNRAS, 167, 31

Font, J. A., Ibáñez, J. M., Marquina, A., \& Martí, J. M. 1994, A\&A, 282, 304

Ghisellini, G., \& Celotti, A. 2001, A\&A, 371, L1

Giacomazzo, B., \& Rezzolla, L. 2006, JFM, 562, 223
Giovannini, G., Cotton, W. D., Feretti, L., Lara, L., \& Venturi, T. 2001, ApJ, 552, 508

Giovannini, G., Taylor, G. B., Feretti, L., et al. 2005, ApJ, 618, 635

Gopal-Krishna, \& Wiita, P. J. 2000, A\&A, 363, 507

Gopal-Krishna, \& Wiita, P. J. 2002, New Astr. Rev., 46, 357

Hardcastle, M. J., Worrall, D. M., Birkinshaw, M., Laing, R. A., \& Bridle, A. H. 2005, MNRAS, 358, 843

Hardee, P. E., Rosen, A., Hughes, P. A., \& Duncan, G. C. 1998, ApJ, 500

Harten, A., Lax, P. D., \& van Leer, B. 1983, on Upstream Differencing and Godunov-Type Schemes for Hyperbolic Conservation Laws, SIAM Rev., 25, 35

Heywood, I., Blundell, K., \& Rawlings, S. 2007, MNRAS, 381, 1093

Hooda, J. S., \& Wiita, P. J. 1996, ApJ, 470, 211

Hooda, J. S., \& Wiita, P. J. 1998, ApJ, 493, 81

Horiuchi, S., Meier, D. L., Preston, R. A., \& Tingay, S. J. 2006, PASJ, 58, 211

Kaiser, C., \& Alexander, P. 1997, MNRAS, 286, 215

Kaiser, C., \& Best, P. 2007, MNRAS, 381, 1548

Kino, M., \& Takahara, F. 2004, MNRAS, 349, 336

Komissarov, S. S., \& Falle, S. A. 1998, MNRAS, 297, 1087

Kraft, R. P., Vázquez, S. E., Forman, W. R., Jones, C., \& Murray, S. S. 2003, ApJ, 592, 129

Krause, M. 2005, A\&A, 431, 45

Keppens, R., Nool, M., Tóth, G., \& Goedbloed, J. P. 2003, Comp. Phys. Commun., 153, 317

Kellermann, K. I., Lister, M. L., Homan, D. C., et al. 2004, ApJ, 609, 539

Laing, R., \& Bridle, A. 2002, MNRAS, 336, 1161

Laing, R. A., Parma, P., \& de Ruiter, H. R. 1999, MNRAS, 306, 513

Laing, R. A., Canvin, J. R., Bridle, A. H., \& Hardcastle, M. J. 2006, MNRAS, 372,510

Loken, C., Burns, J. O., Norman, M. L., \& Clarke, D. 1993, ApJ, 417, 515

Martí, J. M., \& Müller, E. 1994, J. Fluid. Mech., 258, 317

Martí, J. M., \& Müller, E. 2003, Living Rev. Relativity, 6, 7

Martí, J. M., Müller, E., Font, J. A., Ibáñez, J. M. A., \& Marquina, A. 1997, ApJ, 479,151

Mathews, W. G. 1971, ApJ, 165, 147

Meliani, Z., \& Keppens, R. 2007, A\&A, 467, L41

Meliani, Z., Sauty, C., Tsinganos, K., \& Vlahakis, N. 2004, A\&A, 425, 773

Meliani, Z., Keppens, R., Casse, F., \& Giannios, D. 2007, MNRAS, 376, 1189

Mignone, A., \& Bodo, G. 2005, MNRAS, 364, 126

Mignone, A., \& McKinney, J. C. 2007, MNRAS, 378, 1118

Mignone, A., Plewa, T., \& Bodo, G. 2005, ApJS, 160, 199

Mirabel, I. F., \& Rodríguez, L. F. 1999, ARA\&A, 37, 409

McNamara, B. R., Nulsen, P. E. J., Wise, M. W., et al. 2005, Nature, 433, 45

Norman, M. L., Burns, J. O., \& Sulkaen, M. E. 1988, Nature, 335, 146

Owen, F., Hardee, P. E., \& Cornwell, T. J. 1989, ApJ, 340, 698

Owen, F. N., Eilek, J., \& Kassim, N. E. 2000, ApJ, 543, 611

Parker, E. N. 1960, ApJ, 132, 175

Perucho, M., \& Martí, J. M. 2007, MNRAS, 382, 526

Pons, J. A., Martí, J. M., \& Müller, E. 2000, J. Fluid Mech., 422, 125

Rawlings, S., \& Saunders, R. 1991, Nature, 349, 138

Rezzolla, L., \& Zanotti, O. 2001, J. Fluid. Mech., 449, 395

Rezzolla, L., Zanotti, O., \& Pons, J. A. 2003, J. Fluid. Mech., 479, 199

Romero, R., Martí, J. M., Pons, J. A., Ibáñez, J. M., \& Miralles, J. A. 2005, J. Fluid. Mech., 544, 323

Rosen, A., \& Hardee, P. E. 2000, ApJ, 542, 750

Rosen, A., Hughes, P. A., Duncan, G. C., \& Hardee, P. E. 1999, ApJ, 516, 729

Rossi, P., Mignone, A., Bodo, G., Massaglia, S., \& Ferrari, A. 2008, A\&A, 488, 795

Sadun, A., \& Morrison, P. 2002, ApJ, 123, 2312

Sari, R., \& Piran, T. 1995, ApJ, 455, L143

Saxton, C. J., Bicknell, G. V., Sutherland, R. S., \& Midgley, S. 2005, MNRAS, 359,781

Scheck, L., Aloy, M. A., Martí, J. M., Gómez, J. L., \& Müller, E. 2002, MNRAS, 331,615

Synge, J. L. 1957, The relativistic Gas (North-Holland Publishing Compagny)

Tavecchio, F., Maraschi, L., Sambruna, R. M., et al. 2004, ApJ, 614, 64

Tavecchio, F., Maraschi, L., Sambruna, R. M., et al. 2006, ApJ, 641, 732

Tóth, G., \& Odstrčil, D. 1996, J. Comput. Phys., 128, 82

Urry, C. M., \& Padovani, P. 1995, PASP, 107, 803

van der Holst, B., \& Keppens, R. 2007, JCP, 226, 925

Wan, L., Daly, R. A., \& Guerra, E. J. 2000, ApJ, 544, 671

Wang, P., Abel, T., \& Zhang, W. 2008, ApJS, 176, 467

Wold, M., Lacy, M., \& Armus, L. 2007, A\&A, 470, 531

Woods, E., \& Loeb, A. 1995, ApJ, 453, 583

Zhang, W., \& MacFadyen, A. I. 2006, ApJS, 164, 255

Zirbel, E. L. 1997, ApJ, 476, 489 


\section{Appendix A: Relativistic hydrodynamics and the equation of state}

The special relativistic hydrodynamic evolution of a perfect fluid is governed by the conservation of the number of particles, and energy-momentum conservation. These two conservation laws can be written as

$\left(\rho u^{\mu}\right)_{\mu}=0, \quad\left(T^{\mu v}\right)_{\mu}=0$,

where $\rho, \boldsymbol{u}=(\gamma, \gamma \boldsymbol{v})$, and $T^{\mu \nu}=\rho h u^{\mu} u^{\nu}+p g^{\mu \nu}$ define, respectively, the proper density, the four-velocity, and the stress-energy tensor of the perfect fluid. Proper density is related to the number density $n$ in the fluid rest frame $\rho=n m_{\mathrm{p}}$, where $m_{\mathrm{p}}$ indicates the particle (proton) rest mass. The definitions involve the Lorentz factor $\gamma$, the fluid pressure $p$, and the relativistic specific enthalpy $h$. For the (inverse) metric $g^{\mu \nu}$, we take the Minkowski metric. Units are taken where the light speed equals unity.

These equations can be written in conservative form involving the Cartesian coordinate axes and the time axis of a fixed "lab" Lorentzian reference frame as

$\frac{\partial U}{\partial t}+\sum_{j=1}^{3} \frac{\partial F^{j}}{\partial x^{j}}=0$.

The conserved variables can be taken as

$U=\left[D=\gamma \rho, \boldsymbol{S}=\gamma^{2} \rho h \boldsymbol{v}, \tau=\gamma^{2} \rho h-p-\gamma \rho\right]^{T}$,

and the fluxes are then given by

$F=\left[\rho \gamma \boldsymbol{v}, \gamma^{2} \rho h \boldsymbol{v} \boldsymbol{v}+p \mathbf{I}, \gamma^{2} \rho h \boldsymbol{v}-\gamma \rho \boldsymbol{v}\right]^{T}$,

where $\mathbf{I}$ is the $3 \times 3$ identity matrix. To close this system of equations, we can use the Synge-like equation of state (EOS) for an ideal gas as also used by Meliani et al. (2004), which is a polytropic equation with a corresponding classical polytropic index $\Gamma$ relating

$p=\left(\frac{\Gamma-1}{2}\right) \rho\left(\frac{e}{m_{\mathrm{p}}}-\frac{m_{\mathrm{p}}}{e}\right)$,

where $e=m_{\mathrm{p}}+e_{\mathrm{th}}$ is the specific internal energy including rest mass, and $e_{\mathrm{th}}$ is the specific thermal energy. This specific internal energy is given in function of the pressure and density by rewriting the above equation to

$\frac{e}{m_{\mathrm{p}}}=\frac{1}{\Gamma-1} \frac{p}{\rho}+\sqrt{\left(\frac{1}{\Gamma-1} \frac{p}{\rho}\right)^{2}+1}$.

The relativistic specific enthalpy is then given by

$h=\frac{1}{2}\left((\Gamma+1) \frac{e}{m_{\mathrm{p}}}-(\Gamma-1) \frac{m_{\mathrm{p}}}{e}\right)$.

The sound speed (in light speed units) is then found from

$$
\begin{aligned}
c_{\mathrm{s}}^{2} & =\frac{1}{h} \frac{\mathrm{d} p}{\mathrm{~d} \rho} \\
& =\frac{1}{h} \frac{p}{\rho}\left(\frac{(\Gamma+1)}{2}+\frac{(\Gamma-1)}{2} \frac{m_{\mathrm{p}}^{2}}{e^{2}}\right) .
\end{aligned}
$$

At each time step in the numerical integration, the primitive variables $(\rho, \boldsymbol{v}, p)$ involved in flux expressions should be derived from the conservative variables $U$ resulting in a system of nonlinear equations. One can bring this system into a single equation for the pressure $p$, directly following from the definition of the conserved variable $\tau$ from

$(\tau+p+D)\left(1-v(p)^{2}\right)-\rho h(p)=0$,

which yields $\boldsymbol{v}=\boldsymbol{S} /(\tau+p+D)$, once solved for $p$. One inserts $h$ from Eq. (A.7), in which one uses Eq. (A.6), with in addition $D=\gamma \rho$ while $1 / \gamma^{2}=1-S^{2} /(\tau+p+D)^{2}$. This nonlinear Eq. (A.9) is solved using a Newton-Raphson algorithm. For a chosen fixed index $\Gamma=5 / 3$, we then achieve a locally varying, effective polytropic index, taking on values between its relativistic $4 / 3$ and classical 5/3 extremes, found from

$\Gamma_{\mathrm{eff}}=\Gamma-\frac{\Gamma-1}{2}\left(1-\frac{m_{\mathrm{p}}^{2}}{e^{2}}\right)$.

This provides an excellent approximation to the true Synge gas expression, while avoiding costly Bessel function evaluations. If $1<\Gamma \leq 5 / 3$, we incorporate effects ranging from nearisothermal conditions to adiabatic flow, and if $\Gamma>5 / 3$ we incorporate the effect of energy losses.

\section{Appendix B: Numerical tests for realistic EOS}

The main aim of this appendix is to point out that we have determined the exact solutions to newly selected Riemann problems with a realistic equation of state. This exact solution is used here, and can be used by other authors, to benchmark codes. We test the code for adiabatic EOS Riemann problems, as also investigated by Mignone \& McKinney (2007), and for a more general case with equivalent classical polytropic index $3 / 2$ to mimic the heating in a relativistic fluid (Meliani et al. 2004). In each test, we analyse the effect of the equation of state with variable effective polytropic index on the behaviour of the shock and rarefaction waves. We include tests with high Lorentz factor 100, with the aim of investigating shocks in gamma ray bursts, where the forward shock is always relativistic and the reverse shock Newtonian. The matter shocked by the forward shock then has an effective polytropic index reaching 4/3. However, the matter shocked by the reverse shock has the effective polytropic index $5 / 3$. This difference state of the matter between the two shocked media induces a change in the propagation speed of the shocks. In fact, using this EOS will improve the simulations for GRBs and induce some variation from the results in Meliani et al. (2007).

\section{B.1. Exact solution to Riemann problems with realistic EOS}

The solution of the one-dimensional Riemann problem in hydrodynamics consists of determining the temporal evolution of a fluid which, at some initial time, has two adjacent uniform states characterised by different values of uniform velocity, pressure, and density. These initial conditions completely determine the way in which the discontinuity will decay after removal of the barrier separating the initial "left" and "right" states.

In general, the Riemann problem requires the solution of a nonlinear algebraic system of equations written as a function of a set of unknown quantities. In relativistic hydrodynamics (RHD), an exact solution has been obtained only rather recently and was proposed by Martí \& Müller (1994) for flows that are purely along the direction normal to the initial discontinuity. This work has then been extended to the case in which tangential velocities are present (Pons et al. 2000) and improved in efficiency by exploiting the relativistic invariant relative velocity between 

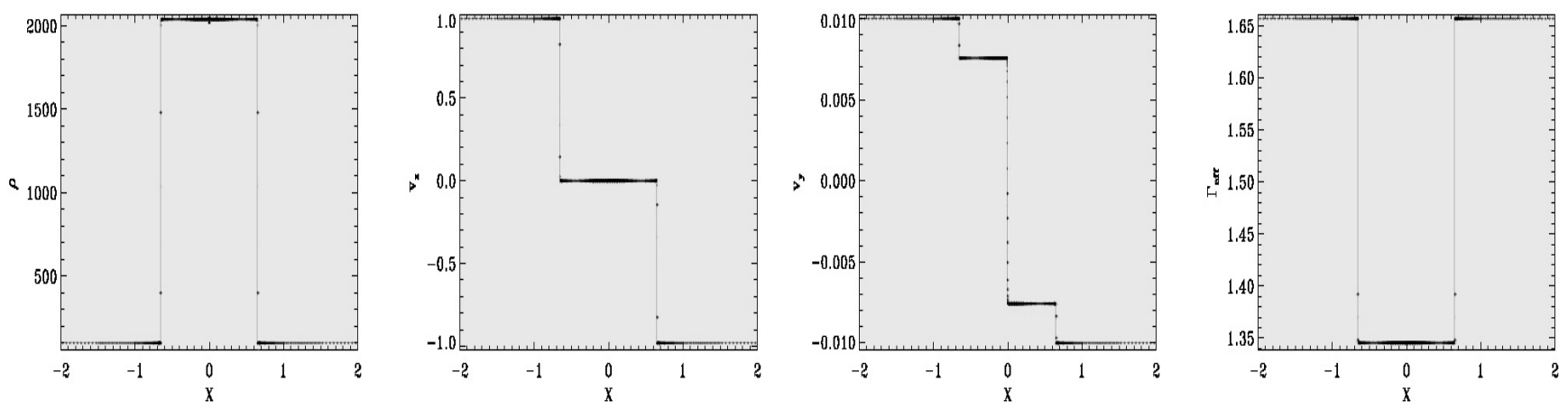

Fig. B.1. One-dimensional Riemann problem for colliding, identical cold flows, with a jump in tangential velocity $v_{y}$. The solid lines are the exact solution. The result is plotted at $t=2$.

the two states to predict the wave pattern produced (Rezzolla \& Zanotti 2001; Rezzolla et al. 2003).

The exact solution is found after expressing all of the quantities behind each wave as functions of the value of the unknown gas pressure $p$ at the contact discontinuity. In this way, the problem is reduced to the search for the value of the pressure that satisfies the jump conditions at the contact discontinuity. Recently, Giacomazzo \& Rezzolla (2006) have found for the first time (after a first attempt by Romero et al. 2005 limited to a very special case) a general procedure to compute the exact solution of the Riemann problem in relativistic magnetohydrodynamics (RMHD). This solver can also be used in RHD by setting the magnetic field to zero and in this case it is similar to the method developed by Pons et al. (2000). Moreover, the exact solver can implement, both in RHD and in RMHD, various equations of state of the form $p=p\left(\rho, e_{\mathrm{th}}\right)$ making it a very general tool that has been used for testing several special and general relativistic numerical codes. To the best of our knowledge, this is the first time in which exact solutions with generic initial states have been computed with a different than polytropic EOS.

\section{B.2. Numerical schemes}

We include in this section various stringent tests to validate the code. We perform one-dimensional test problems to show the code's ability to resolve Riemann problems, and show that we reproduce the exact wave patterns in special relativistic hydro with varying EOS. We use different discretisation schemes, namely TVDLF (Tóth \& Odstrčil 1996), HLLE (Harten et al. 1983; Einfeldt 1988), HLLC (Mignone \& Bodo 2005), and also a hybrid mixture between TVDLF and HLLC. The latter ensures that, in regions where spurious oscillations can be induced by HLLC, the code switches to TVDLF. This switch is effective in cells where the fluxes as computed at the left and right edges change direction. For the linear reconstruction from cell centre to edge, we use a robust minmod scheme that reduces any spurious numerical oscillations. All these $1 \mathrm{D}$ tests are compared to their exact solution, computed as outlined in Sect. B.1.

\section{B.3. $1 D$ tests: Riemann problems}

We starte with the collision of two cold flows with, in addition, an opposite orientation of tangential velocity. The initial states are $\rho_{\mathrm{L}}=100, p_{\mathrm{L}}=1, v_{\mathrm{x}, \mathrm{L}}=5 / \sqrt{26}, v_{\mathrm{y}, \mathrm{L}}=0.01$ (left), and $\rho_{\mathrm{R}}=100, p_{\mathrm{R}}=1, v_{\mathrm{x}, \mathrm{R}}=-5 / \sqrt{26}, v_{\mathrm{y}, \mathrm{R}}=-0.01$ (right). Both flows had a Lorentz factor $\gamma \sim 5$ and the classical polytropic index 5/3. The simulation was done with 20 cells at the lowest grid level, and we allowed for 7 levels on the spatial range $-2<x<2$. The test shows the characteristic pattern of two shock waves and a stationary discontinuity in the tangential velocity. The two shocks convert the kinetic energy to thermal energy and the effective polytropic index, which was $\Gamma_{\text {eff }}=1.657$ throughout drops locally to 1.345 increasing the compression rate $\rho_{\mathrm{C}} / \rho_{\mathrm{L}} \sim 20.44$, where $\rho_{\mathrm{C}}$ denotes the central proper density. In Fig. B.1, we plot the result at $t=2$ as obtained with the hybrid version of HLLC. This scheme captures the two shock waves accurately, as they are propagating with a shock speed $v_{\mathrm{sh}} \sim 0.33$, and seperate the two regions with different states of the matter. Indeed, a classical fluid exists in front of the shock waves and an ultra-relativistic state is found between the shock waves.

In the second test, we followed the evolution of an initial discontinuity between two fluids with different thermodynamic states, both moving to the right. To the right, the relativistic fast gas is characterised by $p_{\mathrm{R}}=5000, \rho_{\mathrm{R}}=0.01, v_{\mathrm{X}, \mathrm{R}}=$ $0.5, v_{\mathrm{y}, \mathrm{R}}=0.5$, and this interacts with the cold slower gas at left $p_{\mathrm{L}}=0.01, \rho_{\mathrm{L}}=1, v_{\mathrm{x}, \mathrm{L}}=0.1, v_{\mathrm{y}, \mathrm{L}}=0.5$, thereby creating a flow to the left. We took $\Gamma=5 / 3$, and obtained on the left an effective polytropic index $\Gamma_{\text {eff, } \mathrm{L}}=5 / 3$, and on the right, it was $\Gamma_{\text {eff, } \mathrm{R}}=4 / 3$. The simulation is done with 20 cells at the lowest grid level, and we allowed for 9 levels on the spatial range $-2<x<2$. The evolution leads to the formation of a shock wave propagating to the left, which heats and compresses the fluid. Between this shock and the contact discontinuity, the effective polytropic index of the compressed fluid drops to 1.45. In Fig. B.2, we plot the result at $t=1$ as obtained with the hybrid version of HLLC. The compression rate $\rho_{\mathrm{L}, \mathrm{s}} / \rho_{\mathrm{L}} \sim 6.69$, where $\rho_{\mathrm{L}, \mathrm{s}}$ denotes the density of the compressed fluid. More to the right of the contact, a rarefaction wave propagates into the relativistic fast fluid. A numerical difficulty was produced by the strong drop in the mass flux, even though the tangential velocity increased at the contact discontinuity, such that the Lorentz factor between the contact discontinuity and the tail of the rarefaction wave is 4.63 higher than in front of the contact discontinuity (see Fig. B.2). In this test, it was vital to introduce a realistic equation of state with effective polytropic index becoming function of the temperature. As the shock propagates to the left, it produces a mildly-relativistic adiabatic compression of the flow, where the state of the fluid is described by $4 / 3<\Gamma<5 / 3$. This state cannot be analysed with a classical constant polytropic equation of state.

The third test represents an isolated contact discontinuity with only a jump in the density $\rho$ such that there is also a jump in 

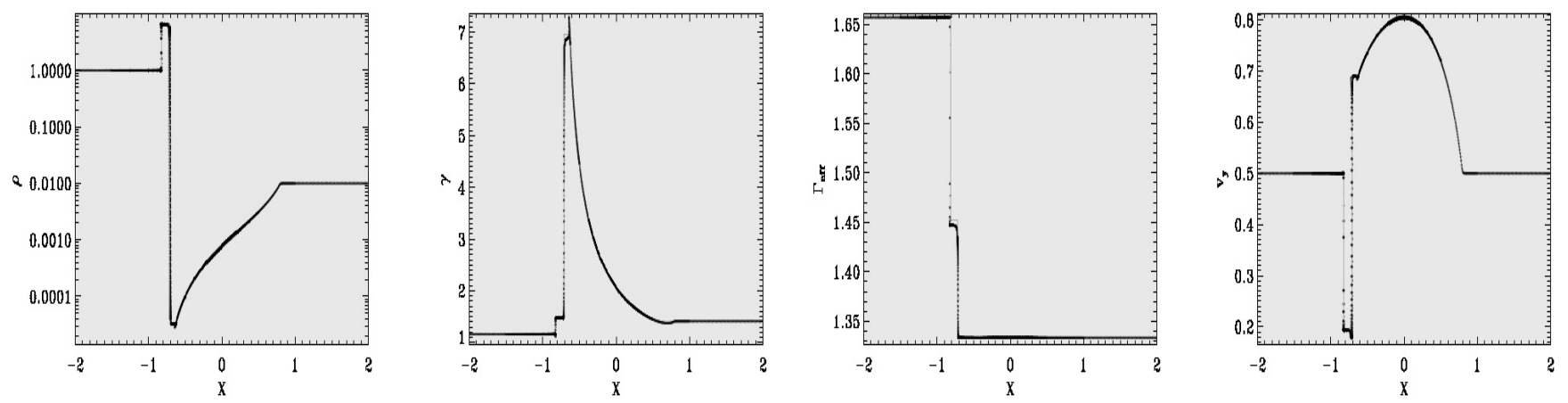

Fig. B.2. One-dimensional Riemann problem for two states with different initial thermodynamic states. The fluid to the left has a classical state and the fluid to the right a relativistic state. The solid lines are the exact solution. The result is plotted at $t=1$.

the effective polytropic index. The initial states were $\rho_{\mathrm{L}}=10^{4}$, $p_{\mathrm{L}}=1.0, v_{\mathrm{x}, \mathrm{L}}=0.0, v_{\mathrm{y}, \mathrm{L}}=0.4($ left $)$, and $\rho_{\mathrm{R}}=0.125, p_{\mathrm{R}}=1.0$, $v_{\mathrm{x}, \mathrm{R}}=0.0, v_{\mathrm{y}, \mathrm{R}}=0.4$ (right). The simulation was done with 12 cells at the lowest grid level, and we allowed for 2 levels on spatial grid $-0.2<x<0.2$. In Fig. B.3, we plot the result (at $t=2$, representative of all times) as obtained with the HLLC scheme. The test shows that HLLC resolves an isolated stationary discontinuity exactly (and thus the isolated jump in effective polytropic index).

In the fourth test, we followed the evolution of an initial discontinuity between two flows to the right, with different Lorentz factor, both having a polytropic index $\Gamma=3 / 2$. This represents the critical value in the Parker model for the solar wind (Parker 1960), and due to our EOS, both flows undergo heating. The initial states were $\rho_{\mathrm{L}}=10.0, p_{\mathrm{L}}=100.0, v_{\mathrm{x}, \mathrm{L}}=0.866025$, $v_{\mathrm{y}, \mathrm{L}}=C_{\mathrm{s}, \mathrm{L}}$ (left), and $\rho_{\mathrm{R}}=1.0, p_{\mathrm{R}}=10^{-3}, v_{\mathrm{x}, \mathrm{R}}=0.30491$, $v_{\mathrm{y}, \mathrm{R}}=C_{\mathrm{s}, \mathrm{R}}$ (right) where $C_{\mathrm{s}}$ denotes the sound speed. The Lorentz factor on the left is already about 100 here. The aim of this test was to mimic the internal shock between a mildly relativistic outflow and subsequent hot and fast ejecta. The simulation was done with 600 cells at the lowest grid level, and we allowed for 13 levels on spatial grid $-2.0<x<2.0$ with a discontinuity at $x=0.0$. In Fig. B.4, we plot the result at $t=0.4$. In this test, we succeeded in resolving the forward shock propagating to the right, the contact discontinuity, and the rarefaction wave propagating to the left (as the fluid in the left is hot). The left propagating rarefaction wave increases the normal speed to $v_{\mathrm{X}, \mathrm{L}} \sim 0.879$, and together with the transverse speed, the Lorentz factor reaches the value $\gamma \sim 233$. This high Lorentz factor in a thin shell between the contact discontinuity and the tail of the rarefaction wave represents a very difficult numerical problem and requires the use of AMR.

\section{B.4. 1D Riemann problem tests for gamma ray bursts}

We now test the ability of the code to model the ultra-relativistic shocks between a relativistic cold flow with a cold external medium. This interaction induces a strong compression of the swept-up matter from the external medium. We modeled three such cases, two with a Lorentz factor of 100 and a third with a Lorentz factor of 10. In all cases, we find a reverse shock, contact discontinuity, and forward shock configuration. In these tests the density of the fast flow is 10 times higher than the density of the stationary medium. All the parameters of the tests are given in Table B.1. These extreme conditions are representative of GRB dynamics, and any code tailored for studying ultra-relativistic flows must be able to compute these situations

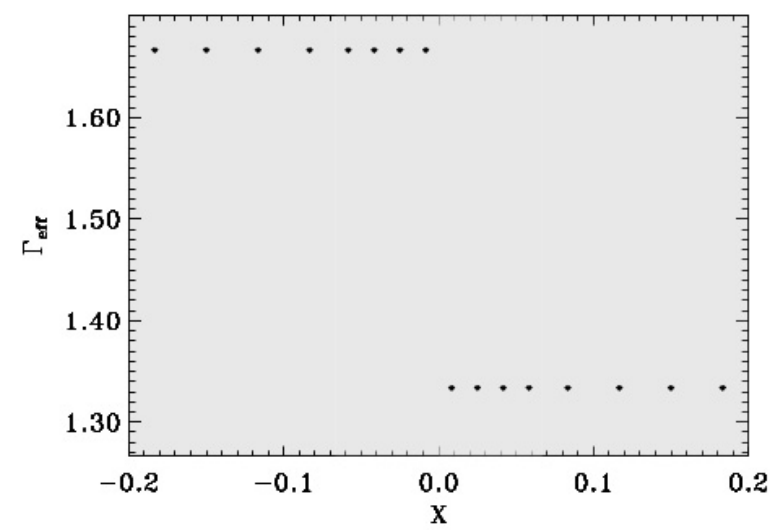

Fig. B.3. Riemann problem for two states with different initial thermodynamic state, with a stationary contact discontinuity at $x=0$.
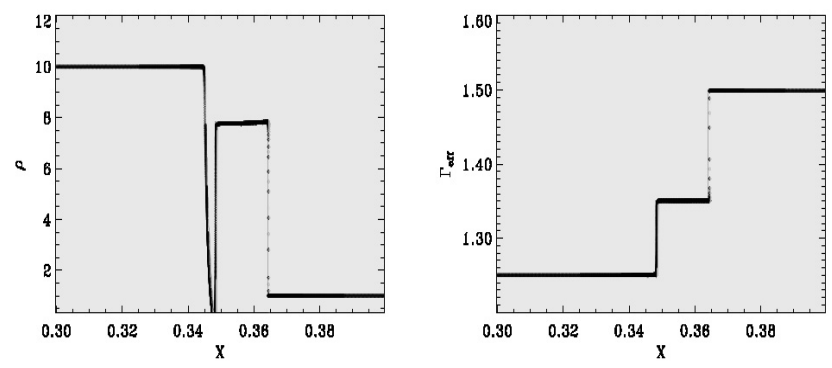

Fig. B.4. One-dimensional Riemann problem for the internal shock in a heated wind, colliding with slower outflow, plotted at $t=0.4$.

accurately. All these test were done with the hybrid version of HLLC. In all these tests there are all four regions that characterise the interaction between an outward moving relativistic beam and the cold external medium: (1) the external medium at rest, (2) the shocked external medium, (3) part of the beam material that is shocked by the reverse shock, (4) unshocked cold material of the beam.

In the first test, the simulation was done with 256 cells at the lowest grid level, and we allowed for 12 levels on the spatial range $-0.1<x<10$ with discontinuity at $x=0.0$, the result at $t=9.5$ is shown in Fig. B.5. The compression rate of the density by the forward shock is $\gamma_{2} n_{2} / n_{1}=\gamma_{2}\left(4 \gamma_{2}+3\right) \sim$ 669.73 , and the thermal energy reaches $e_{2} / m_{p}=\left(\gamma_{2}-1\right) n_{2} \sim$ $616.86 n_{1}$, because the Lorentz factor after the forward shock is 

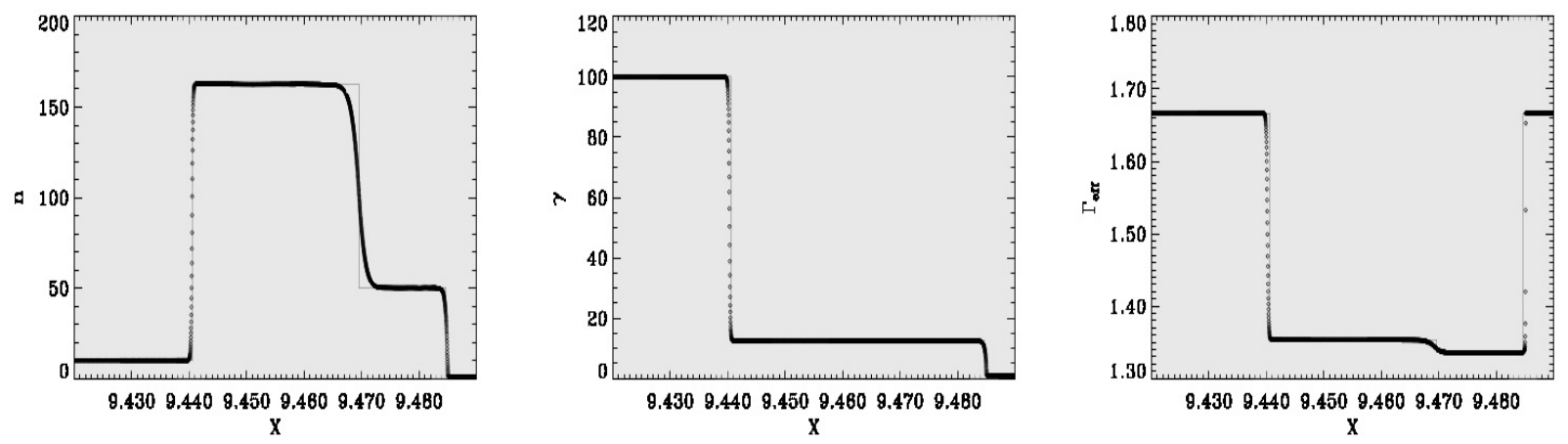

Fig. B.5. GRB test case: a cold Lorentz factor 100 flow penetrates the cold static medium. The solid lines are the exact solution. Left: the density, centre: Lorentz factor, right: effective polytropic index.
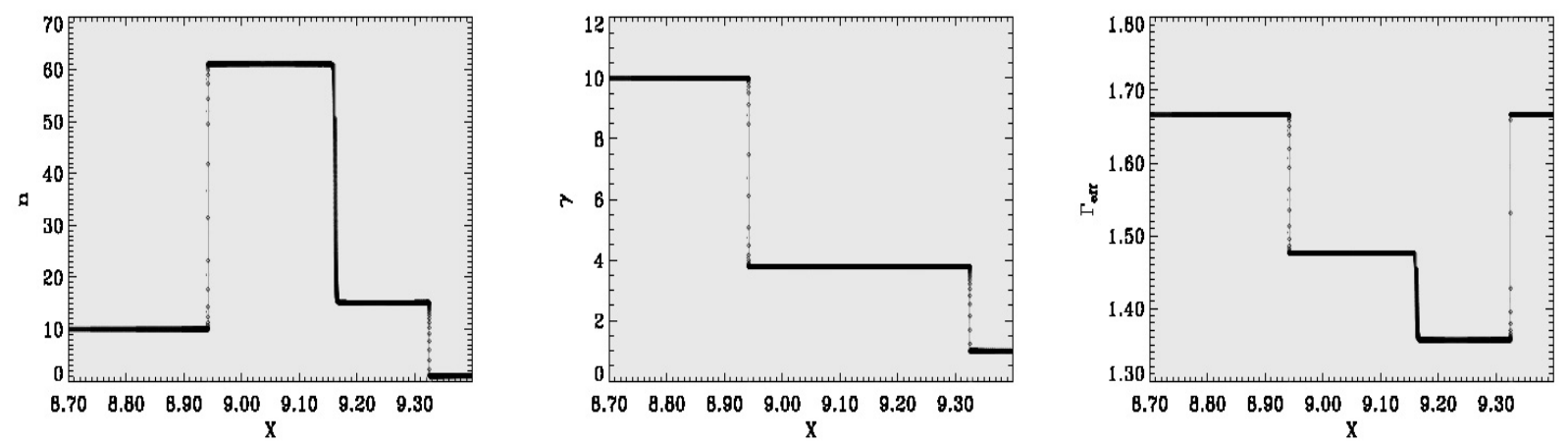

Fig. B.6. Second test case: a cold Lorentz factor 10 flow penetrates into cold static medium. The solid lines are the exact solution. Left: the density, center: Lorentz factor, right: effective polytropic index.

Table B.1. GRB test parameters, the Lorentz factor and the transverse speed of left flow, and the density ratio between left and right state. The fluid in the right is initially at rest.

\begin{tabular}{cccc}
\hline \hline Test & $\gamma_{\mathrm{L}}$ & $\rho_{\mathrm{L}} / \rho_{\mathrm{R}}$ & $v_{y, \mathrm{~L}}$ \\
\hline 1 & 100 & 10 & 0.0 \\
2 & 10 & 10 & 0.0 \\
3 & 100 & 10 & 0.01 \\
\hline
\end{tabular}

$\gamma_{2}=\frac{\gamma_{4}^{1 / 2} f^{1 / 4}}{\sqrt{2}} \sim 12.57$ where $f=\frac{n_{4}}{n_{1}}=10$ (Sari \& Piran 1995). In the region of the shocked swept-up matter, the polytropic index is $4 / 3$ as the thermal energy is higher than the mass energy. Moreover, the reverse shock is propagating with a relative Lorentz factor $\overline{\gamma_{3}} \sim \sqrt{\frac{\gamma_{4}}{2 f^{1 / 2}}} \sim 3.97$, so that the matter is compressed according to $n_{3} / n_{4} \sim\left(4 \overline{\gamma_{3}}+3\right) \sim 18.9$ (the exact obtained value is in fact somewhat lower) and the thermal energy enhanced $e_{3} / m_{p}=\left(\overline{\gamma_{3}}-1\right) n_{3}=56.07 n_{4}$. This implies that the state of the matter is also relativistic and the polytropic index $\Gamma_{3} \sim 4 / 3$. In this case both the forward shock and the reverse shock are relativistic. The numerical difficulty is to resolve the very thin region resulting from the strong compression of sweptup matter, which has an extent of $\Delta X_{2}(t)=0.0015 t$. It is easier to resolve the region between the reverse shock and the contact discontinuity, as the compression by the reverse shock is weaker, and this region extends over $\Delta X_{3}(t)=0.00315 t$.

In a second test, a relativistic cold flow with a Lorentz factor 10 interacts with a cold external medium. This case corresponds more to the interaction of a jet in AGN or micro-quasar with external medium than to GRB case. The simulation was done with 256 cells at the lowest grid level, and we allowed for 8 levels, the result at $t=9.5$ is shown in Fig. B.6. With the weak efficiency of the forward shock $\gamma_{2} \sim 3.79$ to compress the external medium, the thermal energy of swept-up matter increases less and the effective polytropic index reaches a value of $\Gamma_{\text {eff }}=1.356$. The compression rate in this case is lower, hence the swept-up matter has an extent of $\Delta X_{2}(t)=0.0163 t$, which allows the contact discontinuity and the forward shock to be easily resolved. The reverse shock is mildly relativistic with a relative Lorentz factor $\overline{\gamma_{3}} \sim 1.53$ such that the effective polytropic index of the shocked beam only drops to $\Gamma_{\text {eff }}=1.48$. This makes the reverse shock less efficient at compressing the beam matter (compared to the case of a shock with a lower polytropic index $\Gamma=4 / 3)$, and the region between the reverse shock and contact discontinuity then extends over $\Delta X_{3}(t)=0.0225 t$.

When we compare these two simulations, which differ in the Lorentz factor of the beam, namely 100 versus 10, these tests show the dual numerical difficulty when the Lorentz factor increases: (1) the compression rate enhances and (2) the distance between the forward shock and contact discontinuity and between the reverse shock and contact discontinuity decreases.

The last test is a variant of the first, with the Lorentz factor of the beam 100 and transverse velocity $v_{4, y}=0.01$. Introducing a transverse velocity in the beam increases the numerical difficulty of resolving the contact discontinuity with a jump in both density and transverse speed. The simulation is done with 256 cells at the lowest grid level, and we allow for 12 levels on the spatial range $-0.1<x<10$, the result at $t=9.5$ is shown in Fig. B.7. As a result of the interaction, the transverse velocity in the beam material shocked by the reverse shock increases to 

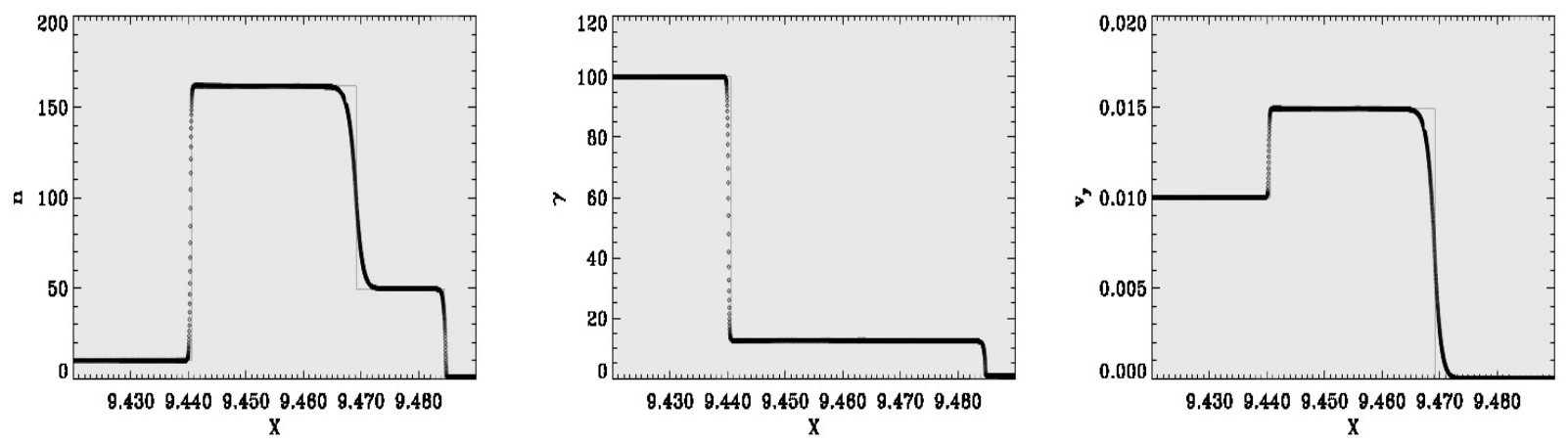

Fig. B.7. GRB test case with transverse velocity: a cold Lorentz factor 100 beam including a weak transverse velocity flow, penetrates the cold static medium. The solid lines are the exact solution. Left: the density, centre: Lorentz factor, right: transverse velocity.

$v_{3, y}=0.015$, which is a purely relativistic effect and depends both on the relativistic kinematics and on the state of the matter (Pons et al. 2000). That mechanism could be significant in the refresh shocks for GRB afterglows, due to the effect of the blastwave meeting a sudden (wind) termination shock in the circumburst medium density profile (Meliani \& Keppens 2007). In more than 1 dimension, such a mechanism will contribute to the shocked shell spreading at a higher speed than the sound speed.

In these tests, it became clear that, in the case when both the forward and reverse shock are relativistic, the constant polytropic index that should be used in simulations that do not have varying effective polytropic index is in fact $4 / 3$. Even if the external medium in these tests is cold, the important polytropic index is that of the shocked fluids. In other cases when the density ratio $n_{4} / n_{1}>\gamma_{4}$ is very high, the reverse shock is near-Newtonian and the effective polytropic index increases to approach $5 / 3$ in the shocked medium between reverse shock and contact discontinuity. Moreover, with a mildlyrelativistic forward or mildly relativistic reverse shock, as in the case with a beam Lorentz factor $\gamma_{4} \leq 10$, the effective polytropic index in swept-up ISM matter is higher than $4 / 3$, while in the shocked-beam matter, the effective polytropic index can be mildly-relativistic as in Fig. B.6 where $\Gamma_{\text {eff }} \sim 1.48$. For all cases, it is more convenient to use the new EOS. 